\title{
Epimerization Reaction of a Substituted Vinyl Cyclopropane Catalyzed by Ruthenium Carbenes: Mechanistic Analysis
}

Xingzhong Zeng, Xudong Wei, * Vittorio Farina, ${ }^{*}$ Elio Napolitano, ${ }^{\natural}$ Yibo Xu, Li

Zhang, Nizar Haddad, Nathan K. Yee, Nelu Grinberg, Sherry Shen, and Chris

Senanayake

Department of Chemical Development, Boehringer Ingelheim Pharmaceuticals, Inc., 900 Ridgebury Road, Ridgefield, CT 06877

'Scuola Normale Superiore, 56100 Pisa, Italy

xwei@rdg.boehringer-ingelheim.com

\section{Supporting Information}

Table of Contents. ..Page

Detailed Experimental Procedures for the Synthesis of

Compounds 5, 6, 8, 9, 11-14, 27a-c and 32.....................................S2-S9

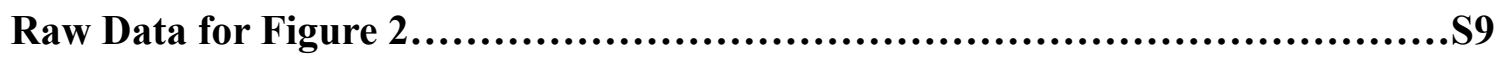

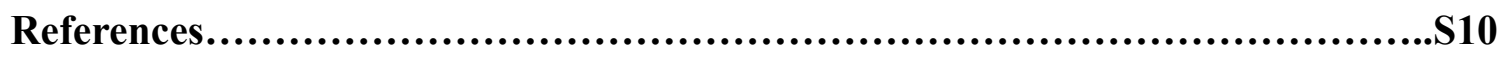

References Truncated in Article.....................................................510

DFT Calculation Supplementary Material.....................................S10-S21

${ }^{1} \mathrm{H}$ and ${ }^{13} \mathrm{C}$ NMR Spectra of

Componds 5, 6, 8, 9, 11-14, 27a-c and 32...................................S22-S43 
$(1 R, 2 S)$-Methyl 1-benzoylamino-2-vinylcyclopropane carboxylate (8). To a suspension of $(1 R, 2 S)$-1-amino-2-vinylcyclopropane carboxylic acid methyl ester tosylate salt $(6.58 \mathrm{~g}, 21 \mathrm{mmol}, 99+\%$ ee) in THF $(100 \mathrm{~mL})$ was added PhCOCl $(2.32 \mathrm{~mL}, 2.81 \mathrm{~g}$, $20 \mathrm{mmol})$ and ${ }^{i} \operatorname{Pr}_{2} \mathrm{NEt}(7.66 \mathrm{~mL}, 5.69 \mathrm{~g}, 44 \mathrm{mmol})$ at RT. The reaction was stirred at RT for 2 hours, diluted with $\mathrm{CH}_{2} \mathrm{Cl}_{2}$, washed with water, dried over $\mathrm{MgSO}_{4}$, filtered and concentrated. Flash chromatography ( silica gel, ethyl acetate $/$ hexanes $=2 / 3$ ) gave the title compound (4.9 g, 100\%) as a white solid. Recrystallization of this material in ethyl acetate led to white needle-like crystals. m.p. $119-121{ }^{\circ} \mathrm{C} ;{ }^{1} \mathrm{H} \mathrm{NMR}\left(400 \mathrm{MHz}, \mathrm{CDCl}_{3}\right) \delta$ $7.78(\mathrm{~d}, J=7.3 \mathrm{~Hz}, 2 \mathrm{H}), 7.4-7.5(\mathrm{~m}, 2 \mathrm{H}), 7.3-7.4$ (m, $2 \mathrm{H})$, 5.7-5.8 (m, $1 \mathrm{H}), 5.25$ (dd, $J$ $=17.1$ and $0.9 \mathrm{~Hz}, 1 \mathrm{H}), 5.09(\mathrm{dd}, J=10.3$ and $1.3 \mathrm{~Hz}, 1 \mathrm{H}), 3.65(\mathrm{~s}, 3 \mathrm{H}), 2.25(\mathrm{q}, J=$ $8.8 \mathrm{~Hz}, 1 \mathrm{H}), 1.91(\mathrm{dd}, J=8.0$ and $5.6 \mathrm{~Hz}, 1 \mathrm{H}), 1.55(\mathrm{dd}, J=9.5$ and $5.6 \mathrm{~Hz}, 1 \mathrm{H}) ;{ }^{13} \mathrm{C}$ NMR $\left(100 \mathrm{MHz}, \mathrm{CDCl}_{3}\right) \delta 170.7,168.1,133.5,133.4,131.7,128.4$ (2 C), 127.1 (2 C), 117.9, 52.3, 40.4, 33.8, 23.1; Anal Calcd for $\mathrm{C}_{14} \mathrm{H}_{15} \mathrm{NO}_{3}$ : C, 68.56; $\mathrm{H}, 6.16$; N, 5.71; found: C, 68.15; H, 5.82; N, 5.24.

$(1 R, 2 R)$-Methyl 1-benzoylamino-2-vinylcyclopropane carboxylate (9). A mixture of $(1 R, 2 S)$-methyl 1-benzoylamino-2-vinylcyclopropane carboxylate (7) (1.96 g, $8 \mathrm{mmol}$ ), Grubbs catalyst $\mathrm{PhCH}=\mathrm{RuCl}_{2}\left(\mathrm{PCy}_{3}\right)_{2}$ (3) (329 mg, $\left.0.4 \mathrm{mmol}\right)$, and $\mathrm{Ph}_{3} \mathrm{P}$ (105 $\mathrm{mg}, 0.4 \mathrm{mmol})$ in toluene $(160 \mathrm{~mL})$ was stirred at $60{ }^{\circ} \mathrm{C}$ for $3 \mathrm{~h}$, cooled down to RT, filtered through Celite, and concentrated. Flash chromatography (silica gel, ethyl acetate / hexanes $=2 / 3)$ gave the title compound $(1.31 \mathrm{~g}, 67 \%)$ as a pale-yellow solid. The starting material (8) was recovered (266 mg, 13.6\%) as a pale-yellow solid. m.p. $108-109{ }^{\circ} \mathrm{C} ;{ }^{1} \mathrm{H}$ NMR (400 MHz, $\left.\mathrm{CDCl}_{3}\right) \delta$ 7.76-7.81 (m, $\left.2 \mathrm{H}\right)$, 7.5-7.55 (m, $\left.2 \mathrm{H}\right)$, 7.4-7.48 (m, $\left.2 \mathrm{H}\right), 6.50$ (s, $1 \mathrm{H}), 5.56-5.68(\mathrm{~m}, 1 \mathrm{H}), 5.32(\mathrm{dd}, J=17.1$ and $0.4 \mathrm{~Hz}, 1 \mathrm{H}), 5.24(\mathrm{dd}, J=10.3$ and $1.1 \mathrm{~Hz}, 1 \mathrm{H}), 3.72(\mathrm{~s}, 3 \mathrm{H}), 2.50(\mathrm{q}, J=8.8 \mathrm{~Hz}, 1 \mathrm{H}), 2.09(\mathrm{dd}, J=9.3$ and $5.6 \mathrm{~Hz}, 1 \mathrm{H})$, $1.42(\mathrm{dd}, J=7.5$ and $5.6 \mathrm{~Hz}, 1 \mathrm{H}) ;{ }^{13} \mathrm{C} \mathrm{NMR}\left(100 \mathrm{MHz}, \mathrm{CDCl}_{3}\right) \delta 172.0,168.4,133.9$, 133.3, 131.4, 128.1 (2 C), 127.0 (2 C), 117.9, 52.3, 38.8, 30.9, 22.0; Anal Calcd for $\mathrm{C}_{14} \mathrm{H}_{15} \mathrm{NO}_{3}$ : C, 68.56; H, 6.16; N, 5.71; found: C, 68.51; H, 6.01; N, 5.62. MS (EI) Cacld. for $\mathrm{C}_{14} \mathrm{H}_{16} \mathrm{NO}_{3}\left[(\mathrm{M}+1)^{+}\right], 246.1130$. Found: 246.1140 .

$(1 R, 2 R)$-Methyl 1-t-butoxycarbonylamino-2-vinylcyclopropane carboxylate

(11): To a solution of $\mathbf{1 0}^{1}(8.33 \mathrm{~g}, 34.52 \mathrm{mmol})$ and triphenylphosphine $(0.27 \mathrm{~g}, 1.04$ 
mmol) in degassed toluene $(3000 \mathrm{~mL})$ at $60{ }^{\circ} \mathrm{C}$, Hoveyda's catalyst $(0.60 \mathrm{~g}, 1.00 \mathrm{mmol})$ was added. The solution was stirred at $60{ }^{\circ} \mathrm{C}$ for 16 hours under nitrogen. The solvent was then removed and the product was purified by flash column chromatography eluted with 3:1 heptane/ethyl acetate. The product was obtained as an off-white solid (5.3 g, 64 \% yield). m.p. $54-56{ }^{\circ} \mathrm{C} .{ }^{1} \mathrm{H}$ NMR $\left(400 \mathrm{MHz}, \mathrm{CDCl}_{3}\right) \delta 5.54(\mathrm{~m}, 1 \mathrm{H}), 5.26(\mathrm{dd}, J=17.2$ $\mathrm{Hz}, J=1.2 \mathrm{~Hz}, 1 \mathrm{H}), 5.17$ (dd, $J=10.4 \mathrm{~Hz}, J=1.2 \mathrm{~Hz}, 1 \mathrm{H}), 3.71(\mathrm{~s}, 3 \mathrm{H}), 2.36(\mathrm{~m}, 1 \mathrm{H})$, $1.90(\mathrm{~m}, 1 \mathrm{H}), 1.45(\mathrm{~s}, 9 \mathrm{H}), 1.29(\mathrm{~m}, 1 \mathrm{H}) ;{ }^{13} \mathrm{C} \mathrm{NMR}\left(100 \mathrm{MHz}, \mathrm{CDCl}_{3}\right) \delta 172.8,156.0$, 133.9, 118.2, 80.0, 52.5, 39.4, 31.3, 28.3, 22.9. Anal. Calcd. for $\mathrm{C}_{12} \mathrm{H}_{19} \mathrm{NO}_{4}$ : C, 59.73; $\mathrm{H}$, 7.94; N, 5.81; found: C, 59.79; H, 8.01; N, 5.60.

$(1 R, 2 R)$-Methyl 1-amino-2-vinylcyclopropane carboxylate tosylate (12): To a solution of 11 (5.3 g, $21.97 \mathrm{mmol})$ in ethyl acetate $(45 \mathrm{~mL}), p$-toluenesulfonic acid monohydrate $(4.18 \mathrm{~g}, 21.97 \mathrm{mmol})$ was added. The mixture was heated to $50{ }^{\circ} \mathrm{C}$ for 2 hours and the solvent was evaporated in vacuo. The residue was dissolved in 10:1 ethyl acetate/methanol and passed through a pad of silica gel. The filtrate was then concentrated, and a brown solid (4.0 g, 58 \% yield) was obtained after crystallization from ethyl acetate. m.p. $140{ }^{\circ} \mathrm{C}$ (decompose). ${ }^{1} \mathrm{H}$ NMR $\left(400 \mathrm{MHz}, \mathrm{DMSO}-d_{6}\right) \delta 8.88$ (br s, 1H), 7.52 (d, J=8.0 Hz, 2 H), 7.14 (d, J=8.0 Hz, 2 H), 5.70 (m, 1 H), 5.48 (d, J=16.4 $\mathrm{Hz}, 1 \mathrm{H}), 5.28$ (d, J=10.8 Hz, $1 \mathrm{H}), 3.71$ (s, $3 \mathrm{H}), 2.44$ (m, $1 \mathrm{H}), 2.29$ (s, $3 \mathrm{H}), 1.76$ (m, $1 \mathrm{H}), 1.56(\mathrm{~m}, 1 \mathrm{H}) ;{ }^{13} \mathrm{C}$ NMR $\left(100.6 \mathrm{MHz}, \mathrm{CDCl}_{3}\right) \delta 169.1,145.0,138.1,131.1,128.2$, 125.5, 121.2, 53.1, 38.4, 28.9, 20.8, 18.5; Anal Calcd for $\mathrm{C}_{14} \mathrm{H}_{19} \mathrm{NO}_{5} \mathrm{~S}$ : C, 53.66; H, 6.11; N, 4.47; found: C, 53.71; H, 5.92; N, 4.29.

Synthesis of (13): A $50 \mathrm{ml}$ round bottom flask was charged with 12 (626 mg, 2 mmol), (1S, 4R) 4-hydroxyproline (462 mg, $2 \mathrm{mmol})$ and DMF (5 mL). $i$-Pr 2 NEt (0.5 $\mathrm{mL}, 0.45 \mathrm{~g}, 3.5 \mathrm{mmol})$ and $\mathrm{EDC}(480 \mathrm{mg}, 2.5 \mathrm{mmol})$ were added at $0{ }^{\circ} \mathrm{C}$, and the reaction mixture was stirred at $0{ }^{\circ} \mathrm{C}$ for 30 minutes and then at room temperature for 1 day. Reaction was quenched with saturated aqueous sodium bicarbonate solution $(15 \mathrm{~mL})$ and extracted with ethyl acetate $(2 \times 20 \mathrm{~mL})$. The combined organic layer was washed with 0.5 $\mathrm{N} \mathrm{HCl}$ solution $(2 \times 15 \mathrm{~mL})$, saturated aqueous sodium bicarbonate solution $(15 \mathrm{~mL})$ and brine $(10 \mathrm{~mL})$. After solvent evaporation, the residue was purified by column chromatography (silica gel, ethyl acetate - hexanes $1: 3$ to $2: 1$ ) to give $\mathbf{1 3}$ as a foam (400 
mg. $56 \%$ yield). ${ }^{1} \mathrm{H}$ NMR (400 MHz, DMSO- $\left.d_{6}\right) \delta 8.41(\mathrm{~s}, 1 \mathrm{H})$ (rotamers: 8.38, 8.35), 5.38-5.55 (m, 1H); 5.15-5.33 (m, 1H), 4.90-5.15 (m, 2H), 4.10-4.32 (m, 2H), $3.59(\mathrm{~s}$, $3 \mathrm{H}), 3.20-3.32(\mathrm{~m}, 2 \mathrm{H}), 2.28-2.45(\mathrm{~m}, 1 \mathrm{H}), 1.95-2.20(1 \mathrm{H}, \mathrm{m}), 1.80-1.95(1 \mathrm{H}, \mathrm{m}), 1.58-$ $1.70(1 \mathrm{H}, \mathrm{m}), 1.35(\mathrm{~s}, 9 \mathrm{H})$ (rotamers: $1.39,1.36) .1 .07-1.17(\mathrm{~m}, 1 \mathrm{H}) .{ }^{13} \mathrm{C} \mathrm{NMR}(100.6$ MHz, DMSO- $d_{6}$ ) $\delta 173.9$ (rotamers: 173.5,173.2); 171.8; 153.6 (rotamers: 153.7, 153.5); 134.6 (rotamers: 135.0; 134.1); 116.8 (rotamers: 117.2, 116.1); 78.5 (rotamer: 78.4); 68.4, 67.6; 58.3 (rotamer: 58.5); 54.5 (rotamers: 54.6, 54.8); 52.1; 38.1 (rotamer: 38.0); 30.3 (rotamer: 30.5), 28.0; 21.6 (21.3). MS (EI) Calcd. for $\mathrm{C}_{17} \mathrm{H}_{27} \mathrm{~N}_{2} \mathrm{O}_{6}\left[(\mathrm{M}+1)^{+}\right] 355.1869$. Found: 355.1871.

Synthesis of (14): To a solution of dipeptide 13 (1.0 g, $2.8 \mathrm{mmol})$ in THF (5 mL) was added triphenylphosphine $(0.89 \mathrm{~g}, 3.38 \mathrm{mmol})$ and 4-nitrobenzoic acid $(0.565 \mathrm{~g}, 3.38$ mmol). The mixture is cooled to $\sim 0^{\circ} \mathrm{C}$ and DIAD $(0.683 \mathrm{~g}, 3.38 \mathrm{mmol})$ in THF $(2 \mathrm{~mL})$ was added dropwise keeping the internal temperature below $5{ }^{\circ} \mathrm{C}$. After the completion of the reaction, the solvent was evaporated and ethyl acetate $(20 \mathrm{~mL})$ was added. The mixture was washed with $2 \% \mathrm{NaHCO}_{3}$ and brine. The organic layer was concentrated to an oil and purified by column chromatography (silica gel, ethyl acetate - hexanes 1:3 to 1:1) to give 14 (988 mg, 70\% yield) as a foam. m.p. $70-74{ }^{\circ} \mathrm{C} .{ }^{1} \mathrm{H}$ NMR $(400 \mathrm{MHz}$, DMSO- $\left.d_{6}, 363 \mathrm{~K}\right) \delta 8.28(2 \mathrm{H}, \mathrm{d}, J=8.9 \mathrm{~Hz}), 8.20$ (d, $\left.J=8.9 \mathrm{~Hz}, 2 \mathrm{H}\right), 7.98$ (br, $\left.1 \mathrm{H}\right), 5.44-$ $5.53(\mathrm{~m}, 2 \mathrm{H}), 5.18(\mathrm{~d}, J=17.2 \mathrm{~Hz}, 1 \mathrm{H}), 5.01(\mathrm{dd}, J=10.4,1.1 \mathrm{~Hz}, 1 \mathrm{H}), 4.35(\mathrm{dd}, J=$ 9.70, $2.5 \mathrm{~Hz}, 1 \mathrm{H}$ ), 3.85 (dd, $J=12.3,5.8 \mathrm{~Hz}, 1 \mathrm{H}$ ), 3.59 (dd, $J=12.3,1.2 \mathrm{~Hz}, 1 \mathrm{H}$ ), 3.45 (s, 3H), 2.68 (ddd, $J=6.1,9.7,14.5 \mathrm{~Hz}, 1 \mathrm{H}), 2.35(\mathrm{~m}, 1 \mathrm{H}), 2.29$ (d, $J=14.5 \mathrm{~Hz}, 1 \mathrm{H})$, $1.65(\mathrm{dd}, J=5.3,9.4 \mathrm{~Hz}, 1 \mathrm{H}), 1.43(\mathrm{~s}, 9 \mathrm{H}), 1.06(\mathrm{dd}, J=5.3,7.1 \mathrm{~Hz}, 1 \mathrm{H}) .{ }^{13} \mathrm{C} \mathrm{NMR}$ $\left(100.6 \mathrm{MHz}, \mathrm{DMSO}-d_{6}, 297 \mathrm{~K}\right) \delta 172.6,171,6,163.9,153.3,150.2,135.2,134.5$ (rotamer: 134.1), 130.9, 123.5 (rotamer: 123.4), 116.8 (rotamer: 117.2), 79.1 (rotamer: 79.3), 74.5, 73.6 (rotamer: 73.8), 58.2, 52.3, 51.9 (rotamer: 51.8), 36.6 (rotamer: 36.3), 31.0, 27.9, 21.3. Anal. Calcd. for $\mathrm{C}_{24} \mathrm{H}_{29} \mathrm{~N}_{3} \mathrm{O}_{9}$ : C, 57.25; H, 5.81; N, 8.35. Found: $\mathrm{C}$, $56.87 ; \mathrm{H}, 5.79 ; \mathrm{N}, 8.35$.

Synthesis of (6): Dipeptide 14 (806 mg, $1.60 \mathrm{mmol}$ ) was treated with $4 \mathrm{~N} \mathrm{HCl}$ in dioxane $(5.0 \mathrm{~mL}, 20.0 \mathrm{mmol})$ at room temperature for $3 \mathrm{~h}$. Solvent and excess $\mathrm{HCl}$ were evaporated directly, yielding a residue which was rinsed with ethyl acetate $(5 \mathrm{~mL})$. This 
gave pure amino acid $\mathrm{HCl}$ salt in almost quantitative yield $(0.70 \mathrm{~g}, 1.59 \mathrm{mmol})$ which was used for the next step directly. To the solid was added dichloromethane $(94 \mathrm{~mL})$ and a solution of $(S)$-2-cyclopentyloxycarbonylamino-8-nonenoic acid $(\mathbf{1 5})^{3}(0.45 \mathrm{~g}, 1.59$ $\mathrm{mmol})$ in dichloromethane $(10 \mathrm{~mL})$, followed by diisopropylethylamine $(0.514 \mathrm{~g}, 3.98$ mmol) at ambient temperature. Solid $O-(1 \mathrm{H}-b e n z o t r i a z o l-1 \mathrm{yl})-N, N, N^{\prime}, N^{\prime}-$ tetramethyluronium tetrafluoroborate (TBTU) $(0.562 \mathrm{~g}, 1.75 \mathrm{mmol})$ was added, and the mixture was stirred for $1 \mathrm{~h}$. The reaction mixture was washed with $2 \% \mathrm{NaHCO}_{3}$ solution and brine $(10 \mathrm{~mL}$ each). After solvent evaporation, the crude product was purified by silica gel column to obtain 6 (0.98 g, 92\%) as a colorless oil. ${ }^{1} \mathrm{H}$ NMR $(600.13 \mathrm{MHz}, 363$ K, DMSO- $d_{6}$ ): $\delta 8.27$ (d, $\left.J=8.9 \mathrm{~Hz}, 2 \mathrm{H}\right), 8.21(\mathrm{~d}, J=8.9 \mathrm{~Hz}, 2 \mathrm{H}), 7.96(\mathrm{br}, 1 \mathrm{H}), 6.58$ (br, $1 \mathrm{H}), 5.70-5.83(\mathrm{~m}, 1 \mathrm{H}), 5.40-5.57(\mathrm{~m}, 2 \mathrm{H}), 5.12-5.25(\mathrm{~m}, 1 \mathrm{H}), 4.80-5.05(\mathrm{~m}, 4 \mathrm{H}), 4.60(\mathrm{t}$, $J=8.0 \mathrm{~Hz}, 1 \mathrm{H}), 4.05-4.30(\mathrm{~m}, 2 \mathrm{H}), 3.70-3.88(\mathrm{~m}, 1 \mathrm{H}), 3.48(\mathrm{~s}, 3 \mathrm{H}), 2.50-2.75(\mathrm{~m}, 1 \mathrm{H})$, 2.25-2.48 (m, 2H), 1.95-2.05 (m, 2H), 1.70-1.85 (m, 2H), 1.45-1.70 (m, 9H), 1.03-1.40 $(\mathrm{m}, 7 \mathrm{H}) .{ }^{13} \mathrm{C}$ NMR $\left(150.92 \mathrm{MHz}, 353 \mathrm{~K}, \mathrm{DMSO}-d_{6}\right): 171.7,171.5,171.2,163.8,156.0$, 150.3, 138.6, 135.2, 134.4, 130.8, 123.5, 116.8, 114.5, 76.2, 74.5, 58.1, 52.4, 52.2, 51.9, 38.0, 33.8, 33.0, 32.3, 30.9, 30.3, 28.3, 28.0, 24.8, 23.2, 21.5. MS (EI) Cacld. for $\mathrm{C}_{34} \mathrm{H}_{45} \mathrm{~N}_{4} \mathrm{O}_{10}\left[(\mathrm{M}+1)^{+}\right]$669.3136. Found: 669.3126.

Synthesis of (5): In a $10 \mathrm{ml}$ flask, $6(27 \mathrm{mg}, 0.04 \mathrm{mmol})$ was dissolved in $5 \mathrm{ml}$ dry toluene, and the solution was purged with argon for $5 \mathrm{~min}$. Hoveyda's catalyst (3.0 $\mathrm{mg}, 0.005 \mathrm{mmol}$ ) was added and the solution was stirred at $60{ }^{\circ} \mathrm{C}$ for $2 \mathrm{~h}$. Solvent was evaporated, and flash chromatography (silica gel, ethyl acetate / hexanes $=2 / 3$ to $3 / 2$ ) gave the title compound (18 mg, 70\%) as a white solid. m.p. 138-140 ${ }^{\circ} \mathrm{C} .{ }^{1} \mathrm{H}$ NMR (500 MHz, $\left.363 \mathrm{~K}, \mathrm{DMSO}_{-} d_{6}\right) \delta 8.27$ (d, $\left.J=8.9 \mathrm{~Hz}, 2 \mathrm{H}\right), 8.21(\mathrm{~d}, \mathrm{~J}=8.9 \mathrm{~Hz}, 2 \mathrm{H}), 7.55$ (s, 1H), 6.40 (br, 1H). 5.22-5.90 (m, 2H), 4.93-5.00 (m, 2H), $4.80(\mathrm{dd}, J=9.22$ and $2.1 \mathrm{~Hz}$, $1 \mathrm{H}), 4.39$ (dt, $J=7.2,3.3 \mathrm{~Hz}, 1 \mathrm{H}), 4.31$ (dd, $J=11.84,6.1 \mathrm{~Hz}, 1 \mathrm{H}), 3.75$ (dd, $J=11.8$, $2.6 \mathrm{~Hz}, 1 \mathrm{H}), 3.42$ (s, 3H), 2.58 (d, $J=14 \mathrm{~Hz}, 1 \mathrm{H}), 2.43$ (ddd, $J=6.3,9.3,15.5 \mathrm{~Hz}, 1 \mathrm{H}$ ), $2.27(\mathrm{q}, J=7.6 \mathrm{~Hz}, 1 \mathrm{H}), 2.15-2.18(\mathrm{~m}, 2 \mathrm{H}), 1.78-1.84(\mathrm{~m}, 3 \mathrm{H}), 1.77(\mathrm{dd}, \mathrm{J}=5.2,9.3 \mathrm{~Hz}$, $1 \mathrm{H}), 1.50-1.75(\mathrm{~m}, 8 \mathrm{H}), 1.20-1.40(\mathrm{~m}, 6 \mathrm{H}) .{ }^{13} \mathrm{C}$ NMR $\left(125.7 \mathrm{MHz}, 363 \mathrm{~K}\right.$, DMSO- $\left.d_{6}\right) \delta$ $171.2,170.9,170.8,163.5,150.1,136.9,134.8,130.3,123.9,122.8,76.1,74.2,57.5$, 
51.8, 51.4, 51.2, 38.0, 31.80, 31.75, 30.5, 28.3, 26.8, 26.6, 24.7, 23.0, 22.6, 21.8. MS (EI) Cacld. for $\mathrm{C}_{32} \mathrm{H}_{41} \mathrm{~N}_{4} \mathrm{O}_{10}\left[(\mathrm{M}+1)^{+}\right]$641.2823. Found: 641.2817.

RCM reaction of $(\boldsymbol{S})$-citronellene. $(S)$-(+)-citronellene (41.5 mg, $0.3 \mathrm{mmol}, 99 \%$ ee) and Grubbs catalyst $\mathrm{PhCH}=\mathrm{RuCl}_{2}\left(\mathrm{PCy}_{3}\right)_{2}(3)(12.3 \mathrm{mg}, 0.015 \mathrm{mmol})$ were added to a dry vial under $\mathrm{Ar} \mathrm{CD}_{2} \mathrm{Cl}_{2}(1.00 \mathrm{~mL}$, degassed with Ar bubbling through at $\mathrm{RT}$ for $1 \mathrm{~h})$ was added to dissolve the two compounds and a purple homogeneous solution resulted, which was transferred to a NMR tube with screw cap and septum under Ar. The sample was monitored by NMR at $303{ }^{\circ} \mathrm{K}\left(500.1 \mathrm{MHz}{ }^{1} \mathrm{H} ; 202.5 \mathrm{MHz}{ }^{31} \mathrm{P}\right)$.

To a solution of $(S)-(+)$-citronellene $\left(138 \mathrm{mg}, 1 \mathrm{mmol}, 99 \%\right.$ ee) in $\mathrm{CH}_{2} \mathrm{Cl}_{2}(50$ $\mathrm{mL}$ ) was added Grubbs catalyst $\mathrm{PhCH}=\mathrm{RuCl}_{2}\left(\mathrm{PCy}_{3}\right)_{2}$ (3) $(41.1 \mathrm{mg}, 0.05 \mathrm{mmol})$. The resultant solution was stirred at RT and monitored by Chiral GC-MS. GC used: HP 6890 with 5973 inert MSD detector. Column: ChiralDex from Astec, $\beta$-Cyclodextrin, Dimethyl; Type: B-DM; Length: 50 meter; Internal Diameter: $0.25 \mathrm{~mm}$; Film thickness: $0.125 \mu \mathrm{m}$. Observed retention time: $61.7 \mathrm{~min}$ for $(+)$-citronellene and $62.5 \mathrm{~min}$ for $(-)$ citronellene.

( \pm ) Methyl 1-benzoylamino-2-deuterium-2-(1-deuteriumvinyl) cyclopropane carboxylate (27a). To $\mathrm{LiAlD}_{4}(80 \mathrm{~mL}, 1.0 \mathrm{M}$ in THF) at RT was added slowly via cannula a solution of 2-butyne-1,4-diol (28) $(5.60 \mathrm{~g}, 65 \mathrm{mmol})$ in THF $(200 \mathrm{~mL})$. [Caution! Gas evolution and exothermic reaction were observed!] After the reaction mixture was stirred at RT for $30 \mathrm{~min}$, it was refluxed $\left(73{ }^{\circ} \mathrm{C}\right)$ for additional $3.5 \mathrm{hrs}$. The reaction mixture was cooled down to $0{ }^{\circ} \mathrm{C}$ and $\mathrm{D}_{2} \mathrm{O}(20 \mathrm{~mL})$ was added dropwise. [Caution! Violent gas evolution and exothermic reaction were observed!] The mixture was filtered, and the cake was washed with ethyl acetate and $\mathrm{MeOH}$. The colorless filtrate solution was dried over $\mathrm{MgSO}_{4}$, filtered, and concentrated. The residue was passed through a pad of silica gel and eluted with ethyl acetate to give 2,3-dideuterium1,4-dideuteroxy-2-butene-1,4-diol (29a) (5.22 g, 87\%) as a colorless oil. ${ }^{1} \mathrm{H}$ NMR (400 $\left.\mathrm{MHz}, \mathrm{CD}_{3} \mathrm{OD}\right) \delta 4.83$ (s, $\left.2 \mathrm{H}\right), 4.03(\mathrm{~s}, 4 \mathrm{H})$.

Bromine $(6.86 \mathrm{~mL}, 21.4 \mathrm{~g}, 0.134 \mathrm{~mol})$ was added dropwise to a suspension of $\mathrm{Ph}_{3} \mathrm{P}(35.2 \mathrm{~g}, 0.134 \mathrm{~mol})$ in $\mathrm{CH}_{3} \mathrm{CN}(170 \mathrm{~mL})$ at $0{ }^{\circ} \mathrm{C}$. The resultant solution was stirred for additional $10 \mathrm{~min}$ at RT, and to this was added a solution of 2,3-dideuterium-1,4- 
dideuteroxy-2-butene-1,4-diol (29a) $(5.15 \mathrm{~g}, 55.9 \mathrm{mmol})$ in $\mathrm{CH}_{3} \mathrm{CN}(110 \mathrm{~mL})$. The resultant orange clear solution was stirred at RT for $3 \mathrm{hrs,} \mathrm{diluted} \mathrm{with} \mathrm{MTBE,} \mathrm{washed}$ with satd $\mathrm{NaHCO}_{3}$ and brine, dried over $\mathrm{MgSO}_{4}$, filtered, and concentrated. Flash chromatography (silica gel, hexanes) gave 2,3-dideuterium-1,4-dibromo-2-butene (30a) (8.89 g, 73.7\%) as white mass. ${ }^{1} \mathrm{H}$ NMR $\left(400 \mathrm{MHz}, \mathrm{CDCl}_{3}\right) \delta 3.95(\mathrm{~s}, 4 \mathrm{H}) ;{ }^{13} \mathrm{C}$ NMR $\left(100 \mathrm{MHz}, \mathrm{CDCl}_{3}\right) \delta 130.3(\mathrm{t}, J=24.2 \mathrm{~Hz}, 2 \mathrm{C}), 30.8(2 \mathrm{C})$.

To a suspension of glycine methyl ester hydrochloride $(6.20 \mathrm{~g}, 49.4 \mathrm{mmol})$ and $\mathrm{MgSO}_{4}(1.41 \mathrm{~g}, 11.8 \mathrm{mmol})$ in $\mathrm{CH}_{2} \mathrm{Cl}_{2}(50 \mathrm{~mL})$ at RT was added PhCHO (4.75 mL, 4.99 $\mathrm{g}, 47 \mathrm{mmol})$ and $\mathrm{Et}_{3} \mathrm{~N}(7.21 \mathrm{~mL}, 5.23 \mathrm{~g}, 51.7 \mathrm{mmol})$ successively. The resultant suspension was stirred at RT overnight. Triethylamine hydrochloride was removed by filtration. The filter cake was washed with $\mathrm{CH}_{2} \mathrm{Cl}_{2}$. The filtrate solution was washed with water, dried over $\mathrm{MgSO}_{4}$, filtered and concentrated below $37{ }^{\circ} \mathrm{C}$ to give the crude imine $\mathrm{PhCH}=\mathrm{NCH}_{2} \mathrm{CO}_{2} \mathrm{Me}(8.21 \mathrm{~g}, 99 \%)$ as a slightly yellow-green oil which was used directly in the next step without further purification. ${ }^{1} \mathrm{H}$ NMR indicated that it is at least $96 \%$ pure with trace amounts of $\mathrm{PhCO}_{2} \mathrm{H} .{ }^{1} \mathrm{H}$ NMR $\left(400 \mathrm{MHz}, \mathrm{CDCl}_{3}\right) \delta 8.25-8.3(\mathrm{~m}, 1$ H), 7.75-7.8 (m, 2 H), 7.76-7.46 (m, 3 H), 4.40 (s, 2 H), 3.76 (s, 3 H).

$\mathrm{LiOBu}^{t}(7.45 \mathrm{~g}, 93.0 \mathrm{mmol})$ was weighed in a glove bag into a $250 \mathrm{~mL}$ flask under $\mathrm{N}_{2}$. $\mathrm{LiBr}$ (4 mg) was added. 2,3-Dideuterium-1,4-dibromo-2-butene (30a) (8.73 g, $40.5 \mathrm{mmol})$ in toluene $(40 \mathrm{~mL})$ was added at RT via cannula and the mixture was stirred at $\mathrm{RT}$ for $5 \mathrm{~min}$. The crude imine $\mathrm{PhCH}=\mathrm{NCH}_{2} \mathrm{CO}_{2} \mathrm{Me}(8.21 \mathrm{~g}, 96 \%, 44.5 \mathrm{mmol})$ in toluene $(30 \mathrm{~mL})$ was added via cannula dropwise to the reaction flask. [Caution! Exothermic reaction!] Lots of $\mathrm{LiBr}$ precipitates formed. The resultant red-orange mixture was stirred at RT overnight, diluted with MTBE, and washed with brine. The red-orange organic layer was used directly in the next step without further purification.

To this red-orange organic layer was added water $(10.5 \mathrm{~mL})$ and $3.75 \%$ aq. $\mathrm{HCl}$ ( $33.7 \mathrm{~mL}, 40.5 \mathrm{mmol}$ ). The orange biphasic mixture was stirred at RT for $1.5 \mathrm{~h}$. The two phases were separated. The aq. phase was charged to a $500 \mathrm{~mL}$ flask and treated with $\mathrm{NaCl}(9.1 \mathrm{~g})$. MTBE $(50 \mathrm{~mL})$ was added. $7.25 \%$ aq. $\mathrm{NaOH}(24.8 \mathrm{~g}, 40.5 \mathrm{mmol})$ was added with stirring to $\mathrm{pH} 9 \sim 10$. The resulted mixture was filtered through Celite to remove a tar-like viscous oil. The biphasic filtrate was separated. The aq. layer was 
extracted once with MTBE. The combined yellow organic layers were dried over $\mathrm{MgSO}_{4}$ and filtered.

To this organic layer was added THF $(23 \mathrm{~mL}), \mathrm{PhCOCl}(4.69 \mathrm{~mL}, 5.7 \mathrm{~g}, 40.5$ mmol) and ${ }^{i} \operatorname{Pr}_{2} \mathrm{NEt}(7.75 \mathrm{~mL}, 5.75 \mathrm{~g}, 44.5 \mathrm{mmol})$. The resultant mixture was stirred at RT overnight and washed with water. The separated aq. layer was extracted once with $\mathrm{CH}_{2} \mathrm{Cl}_{2}$. The combined yellow organic layers were dried over $\mathrm{MgSO}_{4}$, filtered, and concentrated. Flash chromatography (silica gel, ethyl acetate / hexanes $=2 / 3$ ) gave the title compound (27a) (5.25 g, 52\% overall) as a white solid. Recrystallization from ethyl acetate gave needle-like white crystals. m.p. $144-146{ }^{\circ} \mathrm{C} ;{ }^{1} \mathrm{H}$ NMR $\left(400 \mathrm{MHz}, \mathrm{CDCl}_{3}\right) \delta$ 7.76-7.81 (m, 2 H), 7.46-7.52 (m, 1 H), 7.37-7.43 (m, 2 H), 7.14 (bs, 1 H), 5.30 (s, 1 H), $5.14(\mathrm{~s}, 1 \mathrm{H}), 3.69(\mathrm{~s}, 3 \mathrm{H}), 1.95(\mathrm{~d}, J=5.6 \mathrm{~Hz}, 1 \mathrm{H}), 1.61(\mathrm{~d}, J=5.6 \mathrm{~Hz}, 1 \mathrm{H}) ;{ }^{13} \mathrm{C} \mathrm{NMR}$ $\left(100 \mathrm{MHz}, \mathrm{CDCl}_{3}\right) \delta 170.7,168.1,133.7,133.1$ (t, $\left.J=24.2 \mathrm{~Hz}, 1 \mathrm{C}\right), 131.8,128.5$ (2 C), 127.1 (2 C), 117.8, 52.4, 40.3, $33.6(\mathrm{t}, J=24.2 \mathrm{~Hz}, 1 \mathrm{C}), 23.1$; Anal Calcd for $\mathrm{C}_{14} \mathrm{H}_{13} \mathrm{D}_{2} \mathrm{NO}_{3}: \mathrm{C}, 68.00 ; \mathrm{H}, 6.93 ; \mathrm{N}, 5.66$; found: $\mathrm{C}, 67.87 ; \mathrm{H}, 6.70 ; \mathrm{N}, 5.53$.

( \pm ) Ethyl 1-benzoylamino-2-vinylcyclopropane carboxylate (32). The procedure for the preparation of racemic methyl 1-benzoylamino-2-deuterium-2-(1deuteriumvinyl)cyclopropane carboxylate (27a) was followed except that glycine methyl ester hydrochloride was replaced by glycine ethyl ester hydrochloride and 2,3dideuterium-1,4-dibromo-2-butene (30a) was replaced by commercially available (Aldrich) 1,4-dibromo-2-butene. The title compound was obtained as a white solid in $44 \%$ overall yield. m.p. $149.5-151.5{ }^{\circ} \mathrm{C} ;{ }^{1} \mathrm{H}$ NMR $\left(400 \mathrm{MHz}, \mathrm{CDCl}_{3}\right) \delta$ 7.75-7.81 (m, 2 H), 7.47-7.53 (m, $1 \mathrm{H}), 7.38-7.45$ (m, $2 \mathrm{H}), 6.94(\mathrm{bs}, 1 \mathrm{H}), 5.75-5.86(\mathrm{~m}, 1 \mathrm{H}), 5.32$ (dd, $J$ $=17.1$ and $0.9 \mathrm{~Hz}, 1 \mathrm{H}), 5.15(\mathrm{dd}, J=10.3$ and $1.2 \mathrm{~Hz}, 1 \mathrm{H}), 4.1-4.25(\mathrm{~m}, 2 \mathrm{H}), 2.25(\mathrm{q}, J$ $=8.8 \mathrm{~Hz}, 1 \mathrm{H}), 1.98(\mathrm{dd}, J=8.0$ and $5.6 \mathrm{~Hz}, 1 \mathrm{H}), 1.63(\mathrm{dd}, J=9.5$ and $5.6 \mathrm{~Hz}, 1 \mathrm{H})$, $1.22(\mathrm{t}, J=7.1 \mathrm{~Hz}, 3 \mathrm{H}) ;{ }^{13} \mathrm{C}$ NMR $\left(100 \mathrm{MHz}, \mathrm{CDCl}_{3}\right) \delta 170.1,168.1,134.0,133.5$, 131.7, 128.5 (2 C), 127.0 (2 C), 117.9, 61.5, 40.5, 33.8, 23.0, 14.2; Anal Calcd for $\mathrm{C}_{15} \mathrm{H}_{17} \mathrm{NO}_{3}: \mathrm{C}, 69.48 ; \mathrm{H}, 6.61 ; \mathrm{N}, 5.40$; found: $\mathrm{C}, 69.39 ; \mathrm{H}, 6.64 ; \mathrm{N}, 5.33$.

$( \pm)$ Methyl 1-benzoylamino-2-(1-deuteriumvinyl)cyclopropane carboxylate (27b) and ( \pm ) methyl 1-benzoylamino-2-deuterium-2-vinyl cyclopropane carboxylate (27c). The procedure for preparing the compound (27a) was followed except $\mathrm{D}_{2} \mathrm{O}$ was 
replaced by $\mathrm{H}_{2} \mathrm{O}$ to quench the reaction in the first step of the sequence. 2-Deuterium-2butene-1,4-diol (4.80 g, 90\%) was obtained as a colorless oil. ${ }^{1} \mathrm{H}$ NMR $(500 \mathrm{MHz}$, $\left.\mathrm{CD}_{3} \mathrm{OD}\right) \delta 5.81(\mathrm{t}, J=2.0 \mathrm{~Hz}, 1 \mathrm{H}), 4.81(\mathrm{~s}, 2 \mathrm{H}), 4.07(\mathrm{~d}, J=4.1 \mathrm{~Hz}, 4 \mathrm{H}) ;{ }^{13} \mathrm{C} \mathrm{NMR}$ (125 MHz, $\left.\mathrm{CD}_{3} \mathrm{OD}\right) \delta 131.4(2 \mathrm{C}), 63.13,63.09$.

2-Deuterium-1,4-dibromo-2-butene (29b) (12.8 g, 77\%) was obtained as white mass. ${ }^{1} \mathrm{H}$ NMR (500 MHz, $\left.\mathrm{CDCl}_{3}\right) \delta 5.97(\mathrm{t}, J=7.0 \mathrm{~Hz}, 1 \mathrm{H}), 3.95(\mathrm{~d}, J=6.9 \mathrm{~Hz}, 4 \mathrm{H})$; ${ }^{13} \mathrm{C}$ NMR (125 MHz, $\left.\mathrm{CDCl}_{3}\right) \delta 130.7$ (2 C), 30.76, 30.69.

The title equimolar mixture of $( \pm)$ methyl 1-benzoylamino-2-(1deuteriumvinyl)cyclopropane carboxylate (27b) and ( \pm ) methyl 1-benzoylamino-2deuterium-2-vinylcyclopropane carboxylate (27c) $(5.30 \mathrm{~g}, 36.6 \%$ overall) was obtained as a white solid. Recrystallization from ethyl acetate gave needle-like white crystals. ${ }^{1} \mathrm{H}$ NMR (500 MHz, $\left.\mathrm{CDCl}_{3}\right) \delta$ 7.75-7.8 (m, 2 H), 7.47-7.53 (m, 1 H), 7.38-7.44 (m, 2 H), 6.84 (bs, $1 \mathrm{H}), 5.75-5.85(\mathrm{~m}, 0.5 \mathrm{H}), 5.34(\mathrm{~d}, J=16.8 \mathrm{~Hz}, 1 \mathrm{H}), 5.16(\mathrm{dd}, J=8.7$ and 1.5 $\mathrm{Hz}, 1 \mathrm{H}), 3.70$ (s, $3 \mathrm{H}), 2.25$ (q, $J=8.6 \mathrm{~Hz}, 0.5 \mathrm{H}), 1.95-2.0(\mathrm{~m}, 1 \mathrm{H}), 1.6-1.7(\mathrm{~m}, 1 \mathrm{H})$; ${ }^{13} \mathrm{C}$ NMR $\left(125 \mathrm{MHz}, \mathrm{CDCl}_{3}\right) \delta 170.6,168.0,133.5,131.9,128.6(2 \mathrm{C}), 127.1(2 \mathrm{C})$, $118.0,117.9,52.5,34.0,23.26,23.17$.

Table: Raw Data for Figure 2

\begin{tabular}{|c|c|c|c|c|}
\hline \multirow{2}{*}{$\begin{array}{l}\text { Ru cat. / } \\
\mathrm{Ph}_{3} \mathrm{P} \text { (eq) }\end{array}$} & \multicolumn{4}{|l|}{ Ratio of 2:4:5:6 (\%) } \\
\hline & $\mathrm{T}=0.5 \mathrm{~h}$ & $T=2 h$ & $T=6 \mathrm{~h}$ & $\mathrm{~T}=24 \mathrm{~h}$ \\
\hline \multirow{2}{*}{$0.05 / 0.01$} & $49.97 / 43.15 / 4.08 / 2.80$ & $13.49 / 78.31 / 2.77 / 5.43$ & $4.67 / 87.10 / 1.53 / 6.70$ & $* * * *$ \\
\hline & RCM:45.95 Iso: 6.88 & RCM: 83.74; Iso: 8.20 & RCM: 93.80; Iso: 8.23 & $* * * *$ \\
\hline \multirow{2}{*}{$0.05 / 0.03$} & $52.30 / 22.52 / 16.44 / 8.74$ & $13.63 / 47.16 / 17.08 / 22.13$ & $4.85 / 55.42 / 10.22 / 29.50$ & $0.84 / 59.64 / 3.71 / 35.81$ \\
\hline & RCM: 31.26; Iso:25.18 & RCM: 69.29; Iso:39.21 & RCM 84.92; Iso 39.72 & RCM 95.45; Iso 39.52 \\
\hline \multirow{2}{*}{$0.05 / 0.05$} & $71.15 / 9.27 / 13.53 / 6.05$ & $15.96 / 30.28 / 25.81 / 27.95$ & $2.12 / 38.07 / 12.33 / 47.48$ & $0 / 39.50 / 3.84 / 56.66$ \\
\hline & RCM 15.32; Iso: 19.58 & RCM 58.23; Iso 53.76 & RCM 85.55; Iso: 59.81 & RCM 96.16; Iso 60.50 \\
\hline \multirow{2}{*}{$0.05 / 0.10$} & $84.72 / 3.41 / 8.51 / 3.21$ & $38.06 / 15.29 / 29.58 / 17.07$ & $13.36 / 24.33 / 30.50 / 31.81$ & $3.30 / 30.76 / 17.93 / 48.01$ \\
\hline & RCM: 6.62; Iso: 11.72 & RCM: 32.36; Iso 46.65 & RCM 56.14; Iso 62.31 & RCM 78.77; Iso: 65.94 \\
\hline \multirow{2}{*}{$0.05 / 0.20$} & $88.43 / 2.24 / 6.77 / 2.56$ & $58.90 / 8.03 / 22.71 / 10.36$ & $36.01 / 13.59 / 31.42 / 18.98$ & $26.66 / 16.55 / 33.41 / 23.38$ \\
\hline & RCM: 4.80; Iso: 9.33 & RCM 18.39; Iso: 33.07 & RCM: 32.57; Iso:50.40 & RCM: 39.93; Iso:56.79 \\
\hline \multirow{2}{*}{$0.05 / 0.40$} & $96.02 / 0.70 / 2.41 / 0.86$ & $89.89 / 1.93 / 7.40 / 2.58$ & $79.67 / 3.31 / 12.47 / 4.54$ & $75.91 / 4.01 / 14.64 / 5.44$ \\
\hline & RCM: 1.56; Iso: 3.27 & RCM: 4.51; Iso: 9.98 & RCM: 7.85; Iso: 17.01 & RCM: 9.45; Iso: 20.08 \\
\hline \multirow{2}{*}{$0.05 / 1.0$} & $95.05 / 0.59 / 3.54 / 0.80$ & $* * * *$ & $* * * *$ & $84.03 / 2.14 / 10.94 / 2.88$ \\
\hline & RCM: 1.39; Iso: 4.34 & $* * * *$ & $* * * *$ & RCM: 5.02; Iso: 13.82 \\
\hline
\end{tabular}




\section{References:}

[1] Beaulieu, P.L.; Gillard, J.; Bailey, M.D.; Boucher, C.; Duceppe, J.-S.; Simoneau, B.; Wang, X.-j.; Zhang, L.; Grozinger, K.; Houpis, I.; Farina, V.; Heimroth, H.; Krueger, T.; Schnaubelt, J. J. Org. Chem. 2005, 70, 5869-5879.

[2] Schwab, P.; Grubbs, R. H.; Ziller, J. W. J. Am. Chem. Soc. 1996, 118, 100-110.

[3] Faucher, A.-M.; Bailey, M. D.; Beaulieu, P. L.; Brochu, J.-S. D.; Ferland, J.-M.;Ghiro, E.; Gorys, V.; Halmos, T.; Kawai, S. H.; Poirier, M .; Simoneau, B.; Tsantrizos, Y. S.;

Llinàs-Brunet M. Org. Lett. 2004, 6, 2901-2904.

\section{References Truncated in Article:}

13 Yee, N.K.; Farina, V.; Houpis, I.N.; Haddad, N.; Frutos, R.P.; Gallou, F.; Wang, X.-j.; Wei, X.; Simpson, R.D.; Feng, X.W.; Fuchs, V.; Xu, Y.; Tan, J.; Zhang, L.; Xu, J.; Smith-Keenan, L.; Vitous, J.; Ridges, M.D.; Spinelli, E.M.; Johnson, M.; Donsbach, K.; Nicola, T.; Samstag, W.; Brenner, M. J. Org. Chem. 2006, in press.

${ }^{20}$ Gaussian 98, Revision A.6 by Frisch, M.J.; Trucks, G.W.; Schlegel, H.B.; Scuseria, G.E.; Robb, M.A.; Cheeseman, J.R.; Zakrzewski, V.G.; Montgomery, Jr., J.A.; Stratmann, R.E.; Burant, J.C.; Dapprich, J.M.; Millam, J.M.; Daniels, A.D.; Kudin, K.N.; Strain, M.C.; Farkas, O.; Tomasi, J.; Barone, V.; Cossi, M.; Cammi, R.; Mennucci, B.; Pomelli, C.; Adamo, C.; Clifford, S.; Ochterski, J.; Petersson, G.A.; Ayala, P.Y.; Cui, Q.; Morokuma, K.; Malick, D.K.; Rabuck, A.D.; Raghavachari, K.; Foresman, J.B.; Cioslowski, J.; Ortiz, J.V.; Stefanov, B.B.; Liu, G.; Liashenko, A.; Piskorz, P.; Komaromi, I.; Gomperts, R.; Martin, R.L., Foz, D.J.; Keith, T.; Al-Laham, M.A.; Peng, C.Y.; Nanayakkara, A.; Gonzelez, C.; Challacombe, M.; Gill, P.M.W.; Johnson, B.; Chen, W.; Wong, M.W.; Andres, J.L.; Head-Gordon, M.; Replogle, E.S.; Pople, J.A. (Gaussian Inc., Pittsburgh, PA: 1998). For a practical guide, see: Foresman, J.B.; Frisch, A. Exploring Chemistry with Electronic Structure Methods; Gaussian Inc.: Pittsburgh, 1996.

\section{DFT Supplementary material.}

Coordinates (Angstrom) and energies (Hartree - Temperature 298.150 Kelvin, Pressure 1.00000 Atm) of structures A-F and transition structures $(A \leftrightarrow B)$ * and $(A \leftrightarrow C)$ *

$\begin{array}{rrrr}\text { Structure A } & & & \\ 44 & 1.202101 & 0.106956 & 0.059605 \\ 6 & -0.536768 & -0.480520 & -0.224759 \\ 17 & 1.985218 & 0.617164 & -2.208526 \\ 15 & 2.106247 & -2.134424 & -0.210127 \\ 17 & 1.439746 & -0.046080 & 2.484958 \\ 6 & -1.806782 & 0.136095 & 0.177971\end{array}$




\begin{tabular}{|c|c|c|c|}
\hline 15 & $0.657777 \quad 2.473969$ & 0.298339 & \\
\hline 6 & $-3.032337-0.789110$ & 0.434243 & \\
\hline 6 & $-3.017212 \quad 0.073224$ & -0.805598 & \\
\hline 1 & $-0.676917-1.429713$ & -0.772903 & \\
\hline 1 & $1.484332-3.019804$ & -1.150728 & \\
\hline 1 & $2.181416-2.998849$ & 0.926867 & \\
\hline 1 & $3.455209-2.215877$ & -0.675493 & \\
\hline 1 & $-1.755096 \quad 1.015139$ & 0.811449 & \\
\hline 1 & $0.025795 \quad 2.964602$ & 1.485235 & \\
\hline 1 & $-0.132294 \quad 3.130452$ & -0.697484 & \\
\hline 1 & $1.805234 \quad 3.327589$ & 0.277442 & \\
\hline 1 & $-3.676810-0.510772$ & 1.262157 & \\
\hline 1 & $-2.879262-1.858601$ & 0.323401 & \\
\hline 1 & $-2.848808-0.401668$ & -1.767343 & \\
\hline 1 & $-3.652692 \quad 0.952759$ & -0.843496 & \\
\hline Electronic & c Energy $=-296.397454$ & 4871 (Hartree/1 & (Particle) \\
\hline Zero-point & t correction= & 0.144253 & \\
\hline Thermal cc & orrection to Energy= & & 0.160143 \\
\hline Thermal cc & orrection to Enthalpy & & 0.161088 \\
\hline Thermal cc & orrection to Gibbs Fr & ree Energy= & 0.098274 \\
\hline Sum of el€ & ectronic and zero-poi & int Energies= & -296.253202 \\
\hline Sum of el€ & ectronic and thermal & Energies= & -296.237311 \\
\hline Sum of el€ & ectronic and thermal & Enthalpies $=$ & -296.236367 \\
\hline Sum of el€ & ectronic and thermal & Free Energies= & -296.299181 \\
\hline \multicolumn{4}{|c|}{ Structure B. } \\
\hline 44 & $1.201022 \quad 0.087413$ & 0.049418 & \\
\hline 6 & $-0.690152-0.332542-$ & -0.762843 & \\
\hline 15 & 2.380776 & -0.542135 & \\
\hline
\end{tabular}




$$
\begin{array}{rrrr}
15 & 1.772197 & -2.202038 & 0.610071 \\
6 & -1.898015 & -0.229668 & -0.211858 \\
17 & 2.048682 & -0.367624 & -2.207157 \\
17 & 1.853384 & 0.917437 & 2.233849 \\
6 & -2.760091 & 0.077733 & 0.968627 \\
6 & -3.378764 & -0.395772 & -0.385681 \\
1 & 0.026948 & -0.175408 & 1.040598 \\
1 & -0.565174 & -0.652515 & -1.797082 \\
1 & -0.323846 & 3.054368 & 0.211828 \\
1 & 0.226712 & 2.634591 & -1.870204 \\
1 & 1.740957 & 3.341531 & -0.448230 \\
1 & 1.108629 & -3.245901 & -0.105010 \\
1 & 1.593057 & -2.659988 & 1.952300 \\
1 & 3.132692 & -2.586493 & 0.397222 \\
1 & -2.908284 & 1.116388 & 1.258642 \\
1 & -2.772789 & -0.620809 & 1.803544 \\
1 & -3.923493 & 0.336826 & -0.978344 \\
1 & -3.790406 & -1.402083 & -0.431952
\end{array}
$$

Electronic Energy= $-296.336185941 \quad$ (Hartree/Particle) Zero-point correction $=\quad 0.140434$

Thermal correction to Energy $=\quad 0.156581$

Thermal correction to Enthalpy $=\quad 0.157525$

Thermal correction to Gibbs Free Energy= 0.094127

Sum of electronic and zero-point Energies $=\quad-296.195752$

Sum of electronic and thermal Energies= $\quad-296.179605$

Sum of electronic and thermal Enthalpies= $\quad-296.178661$

Sum of electronic and thermal Free Energies= -296.242059 


$\begin{array}{rrrr}44 & 0.723927 & 0.157146 & 0.046824 \\ 6 & -2.053499 & -0.995330 & 0.539174 \\ 15 & 1.637572 & -1.915502 & -0.845081 \\ 15 & -0.015255 & 2.259166 & 1.011977 \\ 6 & -0.749917 & -1.109918 & 0.848511 \\ 17 & 1.729446 & -0.234816 & 2.236565 \\ 17 & 1.676760 & 1.220649 & -1.932016 \\ 6 & -2.389947 & 0.049616 & -0.495549 \\ 6 & -1.076891 & 0.330396 & -1.230577 \\ 1 & -2.826186 & -1.599596 & 1.018108 \\ 1 & 1.064268 & -2.436278 & -2.048170 \\ 1 & 1.598125 & -3.085481 & -0.025260 \\ 1 & 3.017429 & -1.909153 & -1.216737 \\ 1 & -0.883924 & 2.207359 & 2.145391 \\ 1 & -0.706235 & 3.218957 & 0.205756 \\ 1 & 1.025185 & 3.084011 & 1.538626 \\ 1 & -0.369569 & -1.817358 & 1.584599 \\ 1 & -3.162600 & -0.296583 & -1.200920 \\ 1 & -2.792246 & 0.959364 & -0.019096 \\ 1 & -0.869391 & -0.431800 & -1.988933 \\ 1 & -1.011197 & 1.324497 & -1.678223\end{array}$

Electronic Energy $=-296.378981475$

Zero-point correction $=\quad 0.144666$

Thermal correction to Energy= 0.159824

Thermal correction to Enthalpy $=\quad 0.160768$

Thermal correction to Gibbs Free Energy= 0.101798

Sum of electronic and zero-point Energies= $\quad-296.234315$

Sum of electronic and thermal Energies $=\quad-296.219157$

Sum of electronic and thermal Enthalpies= $\quad-296.218213$ 
Sum of electronic and thermal Free Energies $=\quad-296.277183$

\begin{tabular}{|c|c|c|c|}
\hline 44 & 2.606411 & -0.045726 & 0.416021 \\
\hline 6 & 1.076628 & 0.178691 & -0.607583 \\
\hline 17 & 1.715765 & -0.235060 & 2.674422 \\
\hline 15 & 2.843396 & 2.366324 & 0.675228 \\
\hline 17 & 4.442570 & -0.004475 & -1.196390 \\
\hline 6 & -0.005695 & -0.785244 & -0.872146 \\
\hline 15 & 2.747664 & -2.487418 & 0.322022 \\
\hline 6 & -0.893350 & -0.652026 & -2.132053 \\
\hline 6 & -1.512339 & -0.310062 & -0.797665 \\
\hline 7 & -2.515154 & -1.112621 & -0.141898 \\
\hline 6 & -1.871425 & 1.112125 & -0.416043 \\
\hline 7 & -2.971427 & 0.995297 & 0.428484 \\
\hline 6 & -3.389738 & -0.350564 & 0.621000 \\
\hline 8 & -1.310924 & 2.163854 & -0.780021 \\
\hline 8 & -4.344019 & -0.733110 & 1.315170 \\
\hline 1 & 0.893101 & 1.164044 & -1.056551 \\
\hline 1 & 2.058983 & 3.034100 & 1.665237 \\
\hline 1 & 2.598911 & 3.207204 & -0.456247 \\
\hline 1 & 4.149507 & 2.818518 & 1.036470 \\
\hline 1 & 0.111623 & -1.792660 & -0.482504 \\
\hline 1 & 2.780724 & -3.164290 & -0.938486 \\
\hline 1 & 1.816292 & -3.315946 & 1.026981 \\
\hline 1 & 3.965242 & -2.987756 & 0.880041 \\
\hline 1 & -1.215471 & -1.566001 & -2.622822 \\
\hline 1 & -0.681507 & 0.179012 & -2.798293 \\
\hline 1 & -2.582497 & -2.119883 & -0.175756 \\
\hline
\end{tabular}


1

$$
-3.439999 \quad 1.775281
$$

0.872771

Electronic Energy $=-632.547775877$

$\begin{array}{ll}\text { Zero-point correction }= & 0.176592\end{array}$

$\begin{array}{ll}\text { Thermal correction to Energy }= & 0.197005\end{array}$

$\begin{array}{ll}\text { Thermal correction to Enthalpy }= & 0.197949\end{array}$

Thermal correction to Gibbs Free Energy= $\quad 0.123660$

Sum of electronic and zero-point Energies= 632.371184

Sum of electronic and thermal Energies= 632.350771

Sum of electronic and thermal Enthalpies= 632.349827

Sum of electronic and thermal Free Energies= 632.424116

\section{Structure E}

7

6

7

6

6

44

6

6

6

8

8

17

17

15

15
2.523192

2.484544

1.288563

0.476568

1.385268

$-1.377476$

$-1.822499$

$-1.132319$

0.058829

1.183426

3.346703

0.107703

$-3.781557$

$-1.247257$

$-1.791856$
$-0.456577$

$-0.566538$

0.781484

0.112983

0.762237

0.824551

$-0.406158$

0.576895

$-1.227740$

$-0.342175$

0.289428

$-0.539149$

0.335428

1.508399

$-0.393890$

2.403228

$-1.143175$

1.861641

$-2.387630$

$-0.755351$

1. 677072

0.090781

0.561840

$-2.454658$

0.557568

$-0.700851$

2.744638

$-0.408562$

$-2.078536$

$-1.049041$ 
1

1

1

1

1

1

1

1

1

1

1

1

$\begin{array}{rrr}3.254721 & -0.694680 & -1.224550 \\ 1.054258 & 1.478831 & 1.497788 \\ -2.698371 & 0.924619 & 1.777208 \\ -1.413553 & -0.467447 & 3.454194 \\ 0.903802 & -1.165210 & 2.566512 \\ -0.188455 & -2.186666 & 1.628264 \\ -1.827450 & 3.395175 & -1.537993 \\ -1.929736 & 3.422435 & 0.649676 \\ 0.019546 & 3.404537 & -0.363015 \\ -3.152435 & -2.311612 & -1.413076 \\ -1.601320 & -3.108488 & -0.081697 \\ -1.094515 & -2.619558 & -2.165713\end{array}$

Electronic Energy $=-632.538360387$

Zero-point correction=

0.177636

Thermal correction to Energy=

0.197138

Thermal correction to Enthalpy=

0.198083

Thermal correction to Gibbs Free Energy=

0.129946

Sum of electronic and zero-point Energies= 632.360724

Sum of electronic and thermal Energies= 632.341222

Sum of electronic and thermal Enthalpies= 632.340278

Sum of electronic and thermal Free Energies= 632.408414

Structure F
44
2.189997
$-0.027688$
0.196233
15
1.755168
2.361660
0.509817
0.858758
2.808354
1.521239 


\begin{tabular}{|c|c|c|c|}
\hline 1 & 2.951758 & 3.044257 & 0.888424 \\
\hline 1 & 1.337783 & 3.131332 & -0.609032 \\
\hline 15 & 2.920483 & -2.346628 & -0.076743 \\
\hline 1 & 2.096322 & -3.391182 & 0.451050 \\
\hline 1 & 3.139138 & -2.845754 & -1.397894 \\
\hline 1 & 4.165326 & -2.684104 & 0.536443 \\
\hline 17 & 2.500040 & -0.409486 & 2.575871 \\
\hline 17 & 3.843870 & 0.715993 & -1.423802 \\
\hline 6 & 1.062272 & -0.403478 & -1.509037 \\
\hline 1 & 1.665082 & -0.528640 & -2.407805 \\
\hline 6 & -0.280829 & -0.428640 & -1.543416 \\
\hline 1 & -0.862198 & -0.536876 & -2.459584 \\
\hline 6 & 0.120724 & -0.402497 & 0.857922 \\
\hline 1 & -0.039265 & 0.232359 & 1.731803 \\
\hline 1 & 0.170979 & -1.447252 & 1.180698 \\
\hline 6 & -0.978853 & -0.250502 & -0.218224 \\
\hline 6 & -1.724483 & 1.112647 & -0.111409 \\
\hline 8 & -1.208554 & 2.245551 & -0.151859 \\
\hline 7 & -2.114583 & -1.185802 & -0.017693 \\
\hline 7 & -3.068098 & 0.826898 & 0.063862 \\
\hline 1 & -3.797276 & 1.521981 & 0.169895 \\
\hline 1 & -2.024162 & -2.191993 & -0.049827 \\
\hline 6 & -3.340023 & -0.572946 & 0.131053 \\
\hline 8 & -4.461685 & -1.083722 & 0.290671 \\
\hline
\end{tabular}

Electronic Energy $=-632.536813600$

$\begin{array}{ll}\text { Zero-point correction }= & 0.177257\end{array}$

Thermal correction to Energy= 0.196801

Thermal correction to Enthalpy= 0.197745

Thermal correction to Gibbs Free Energy= $\quad 0.128691$ 
Sum of electronic and zero-point Energies= 632.359557

Sum of electronic and thermal Energies= 632.340012

Sum of electronic and thermal Enthalpies= 632.339068

Sum of electronic and thermal Free Energies= 632.408123

$\begin{array}{crrr}\text { Transition structure } & (\mathrm{A} \leftrightarrow \mathrm{B}) *(\mathrm{H}-\mathrm{sh} \text { ift }) & \\ 6 & 0.000000 & 0.000000 & 0.000000 \\ 6 & 0.000000 & 0.000000 & 1.410156 \\ 6 & 0.715321 & 0.000000 & 2.790385 \\ 6 & -0.676527 & 0.634066 & 2.594840 \\ 44 & 0.805270 & -1.101406 & -1.323894 \\ 15 & 0.373727 & -3.433666 & -0.638571 \\ 17 & 3.222361 & -1.427740 & -1.224077 \\ 15 & 1.213379 & 0.901108 & -2.701304 \\ 17 & -1.202659 & -1.440919 & -2.689161 \\ 1 & -0.568748 & 0.904670 & -0.311318 \\ 1 & 1.973448 & 1.986127 & -2.154610 \\ 1 & 0.091845 & 1.615124 & -3.225915 \\ 1 & 2.018657 & 0.775258 & -3.877004 \\ 1 & 0.433407 & -1.572656 & 1.281943 \\ 1 & -0.987742 & -3.752576 & -0.353605 \\ 1 & 0.998180 & -4.197344 & 0.398128 \\ 1 & 0.640670 & -4.323509 & -1.726981 \\ 1 & -1.573322 & 0.161221 & 2.986926 \\ 1 & -0.688638 & 1.723000 & 2.622959 \\ 1 & 1.573834 & 0.660145 & 2.903346\end{array}$




$$
0.793769
$$$$
-0.922148
$$

3.353421

Electronic Energy= $-296.294577174$

Zero-point correction= 0.136639

Thermal correction to Energy=

0.152479

Thermal correction to Enthalpy= 0.153424

Thermal correction to Gibbs Free Energy= 0.091400

Sum of electronic and zero-point Energies= 296.157938

Sum of electronic and thermal Energies= 296.142098

Sum of electronic and thermal Enthalpies= 296.141154

Sum of electronic and thermal Free Energies= 296.203177

$$
\begin{aligned}
& \text { Transition Structure }(A \leftrightarrow C) \text { * (C-shift) } \\
& \begin{array}{lllll}
44 & -0.555287 & 0.019371 & -0.017730
\end{array} \\
& 6 \quad 2.134514-0.392393-0.806326 \\
& 15-1.252127-2.318270 \quad 0.213930 \\
& \begin{array}{lllll}
15 & -0.663731 & 2.438806 & -0.094486
\end{array} \\
& 6 \quad 0.843570-0.192458-1.332231 \\
& \begin{array}{lllll}
17 & -2.188390 & 0.098454 & -1.850057
\end{array} \\
& \begin{array}{llll}
17 & -1.972215 & 0.359760 & 2.106367
\end{array} \\
& \begin{array}{llll}
6 & 2.593250 & 0.363851 & 0.447987
\end{array} \\
& 6 \quad 1.503853-0.367698 \quad 1.142470 \\
& 12.821218-1.145510-1.203143 \\
& 1 \quad-0.835233-3.083186 \quad 1.350514 \\
& 1 \quad-0.993941-3.269246-0.825416 \\
& 1 \quad-2.665050-2.434031 \quad 0.327248 \\
& 1 \quad-0.198933 \quad 3.156091-1.242804
\end{aligned}
$$




$\begin{array}{lrrr}1 & -0.090228 & 3.247366 & 0.939851 \\ 1 & -2.016984 & 2.872522 & -0.040332 \\ 1 & 0.656992 & -0.291255 & -2.407255 \\ 1 & 3.611791 & 0.092008 & 0.731221 \\ 1 & 2.470354 & 1.446155 & 0.398782 \\ 1 & 1.698019 & -1.369359 & 1.514269 \\ 1 & 0.678986 & 0.190518 & 1.637954\end{array}$

Electronic Energy $=-296.331777202$

Zero-point correction $=\quad 0.141178$

Thermal correction to Energy= $\quad 0.156387$

Thermal correction to Enthalpy= 0.157332

Thermal correction to Gibbs Free Energy= 0.097967

Sum of electronic and zero-point Energies= $\quad-296.190600$

Sum of electronic and thermal Energies= $\quad-296.175390$

Sum of electronic and thermal Enthalpies= $\quad-296.174446$

Sum of electronic and thermal Free Energies= -296.233810 

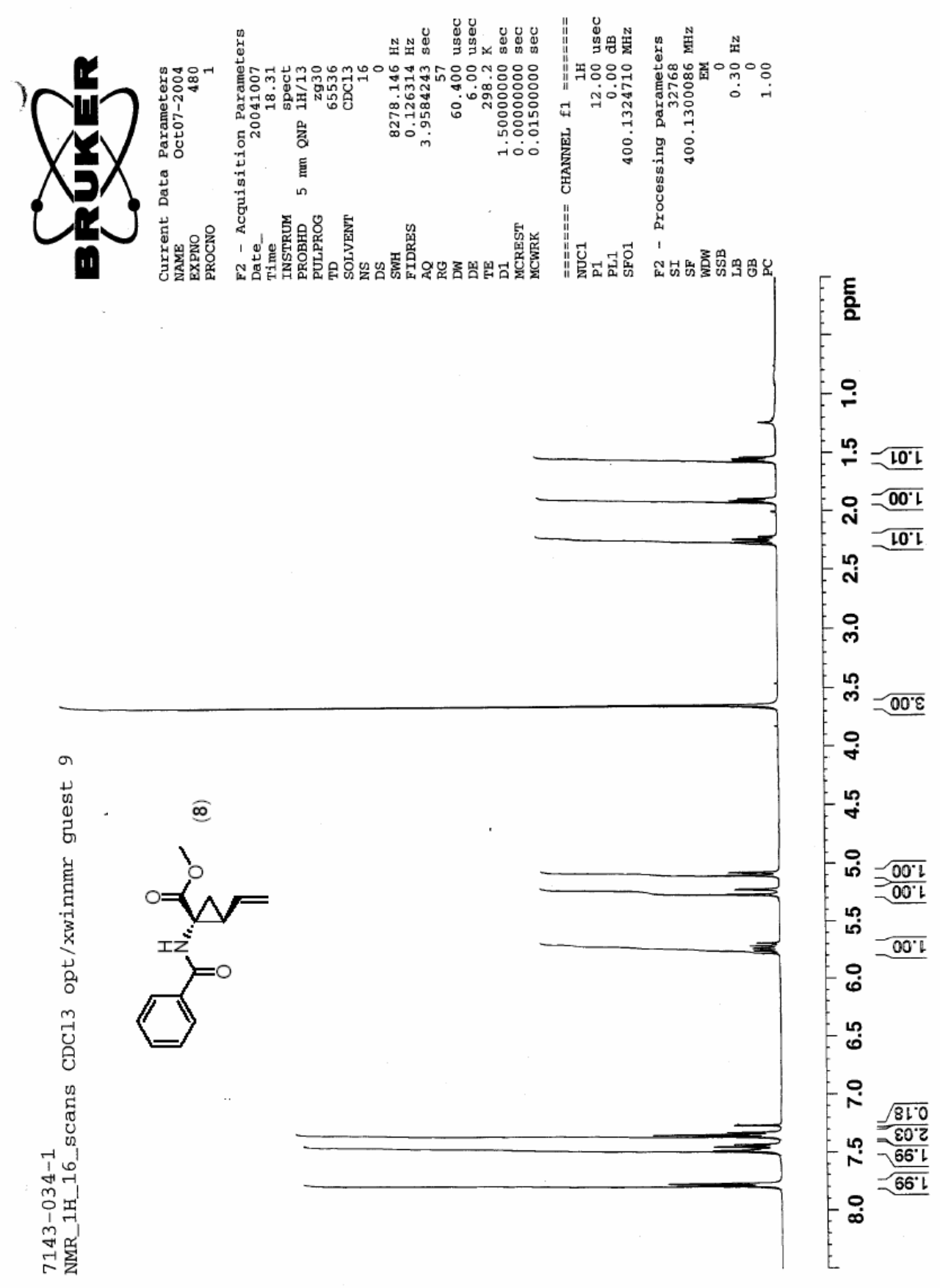

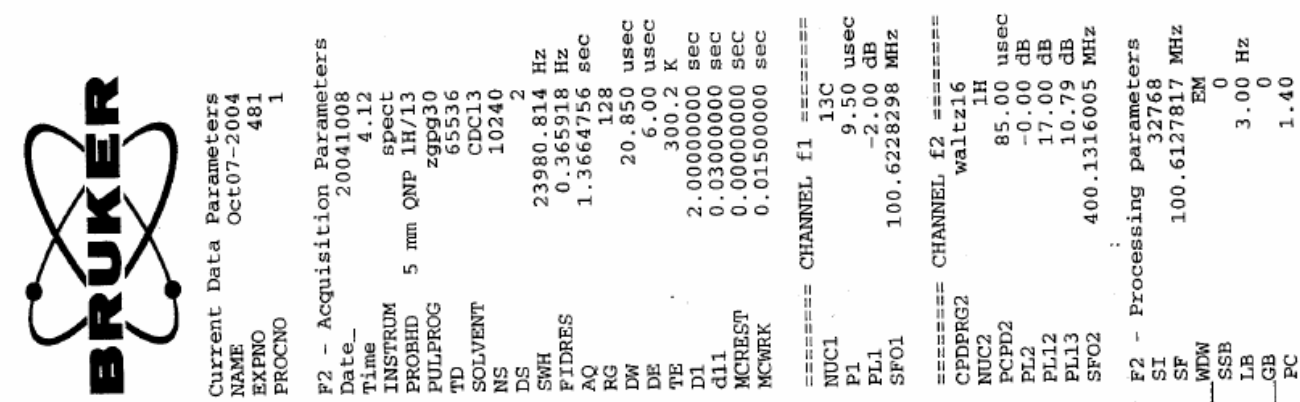

पूल

욕
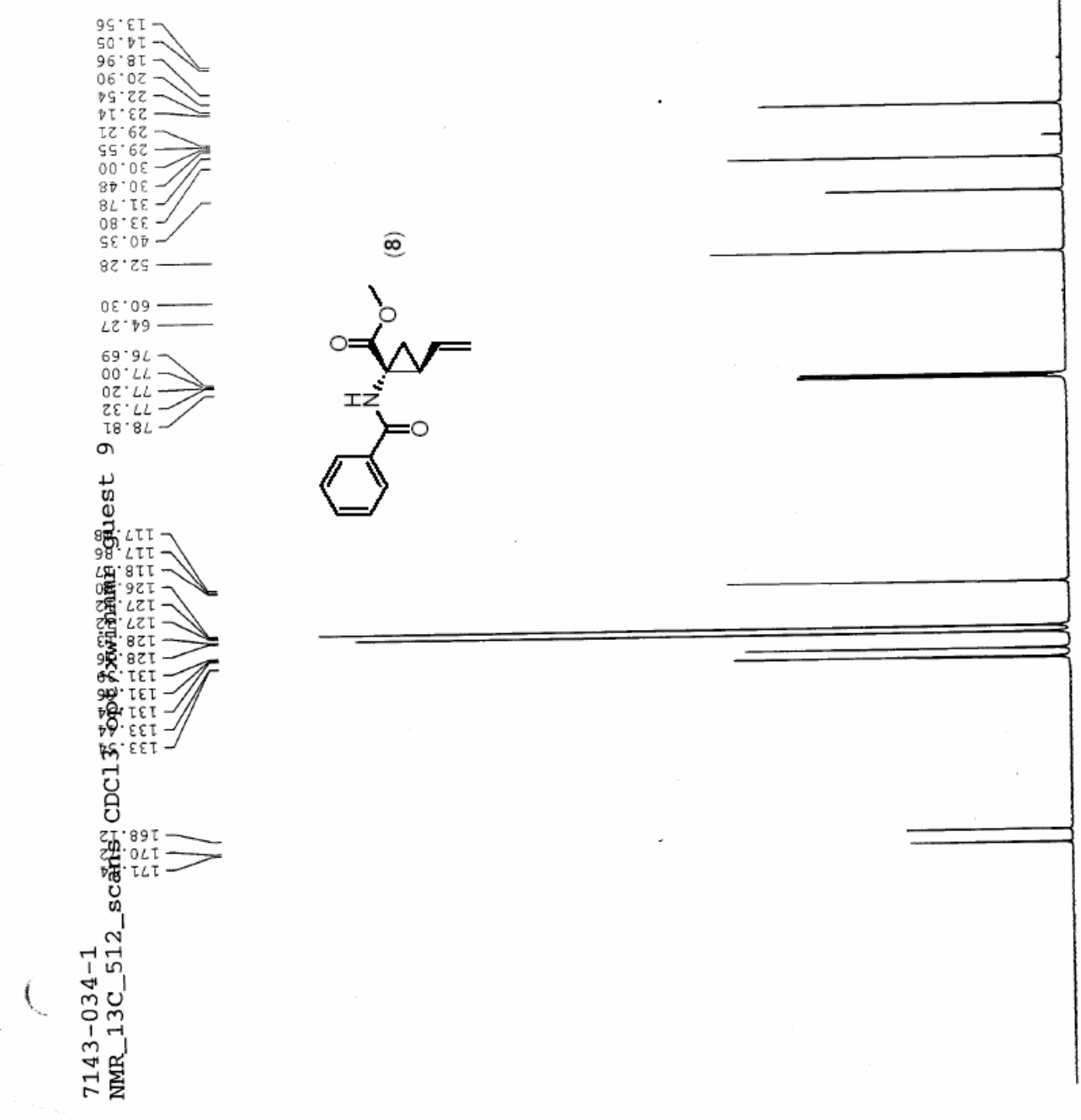

-

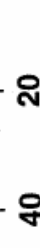

q

8

๕

웅

ิㅗำ

g

8

ळ

จั 

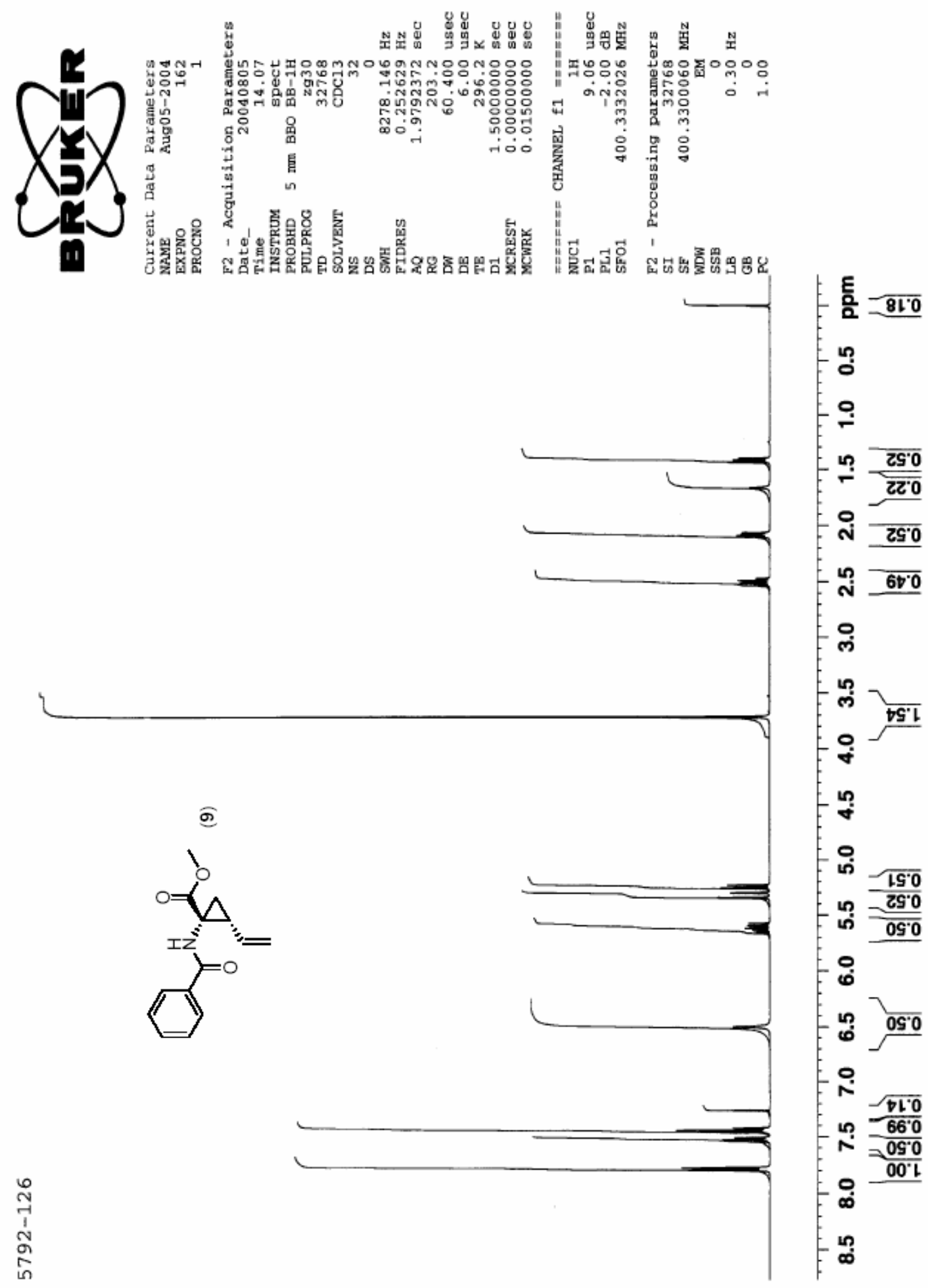


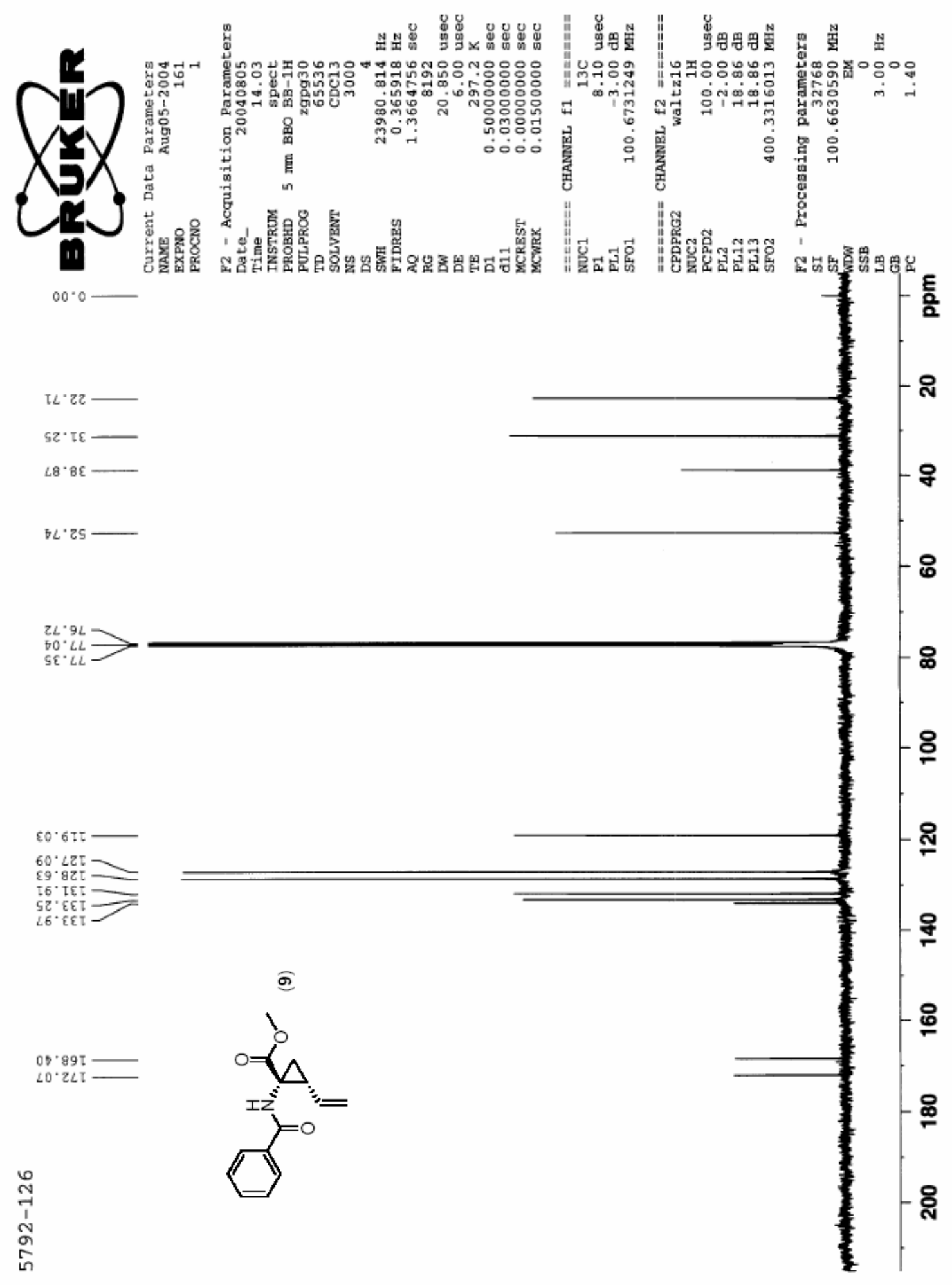



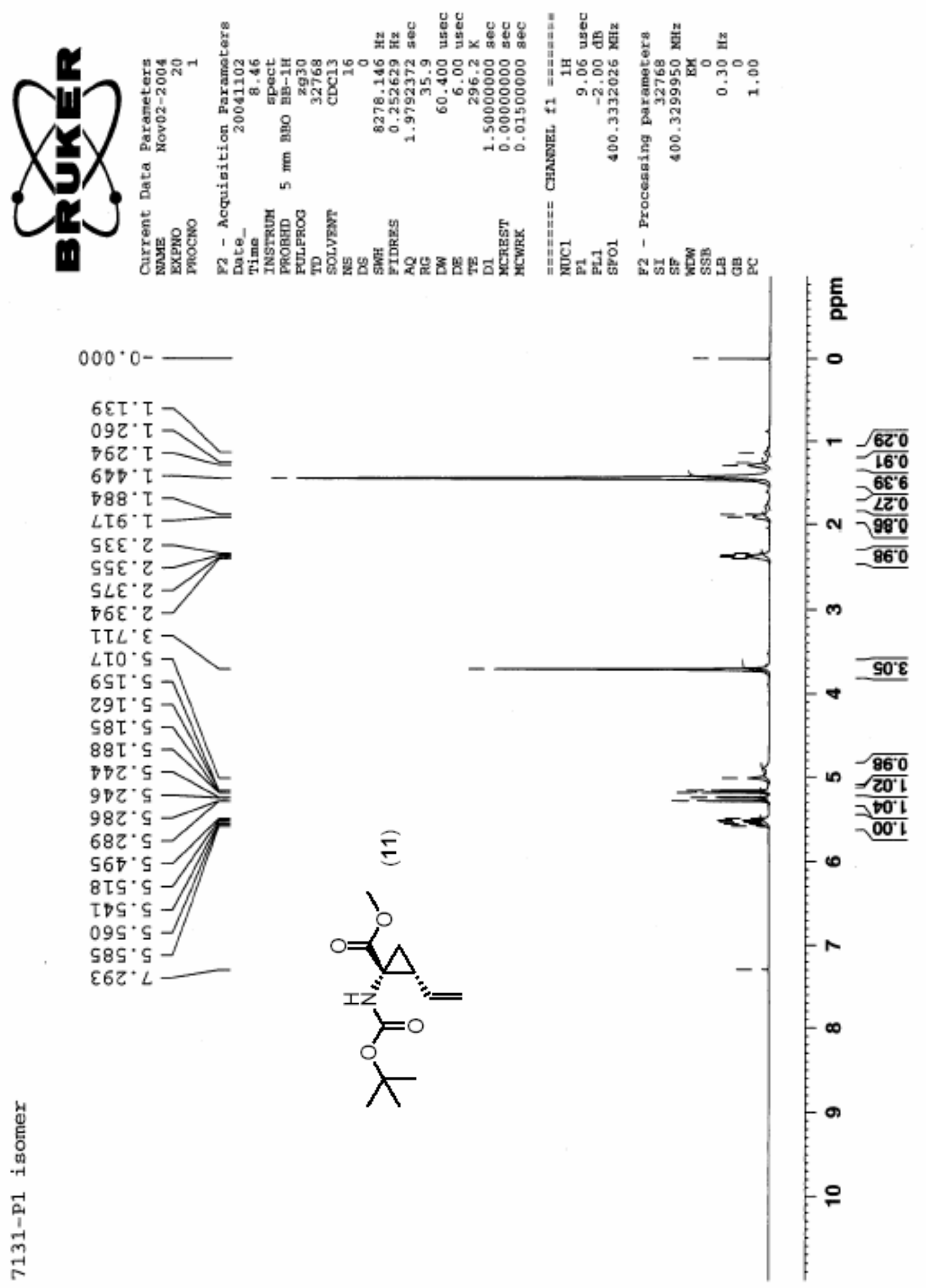


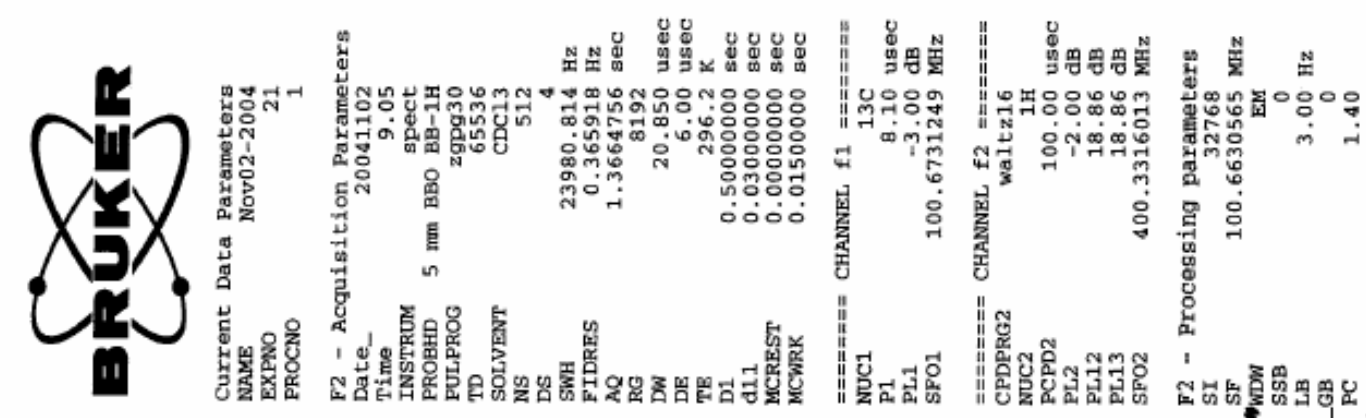

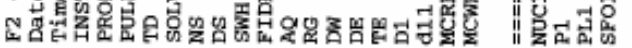



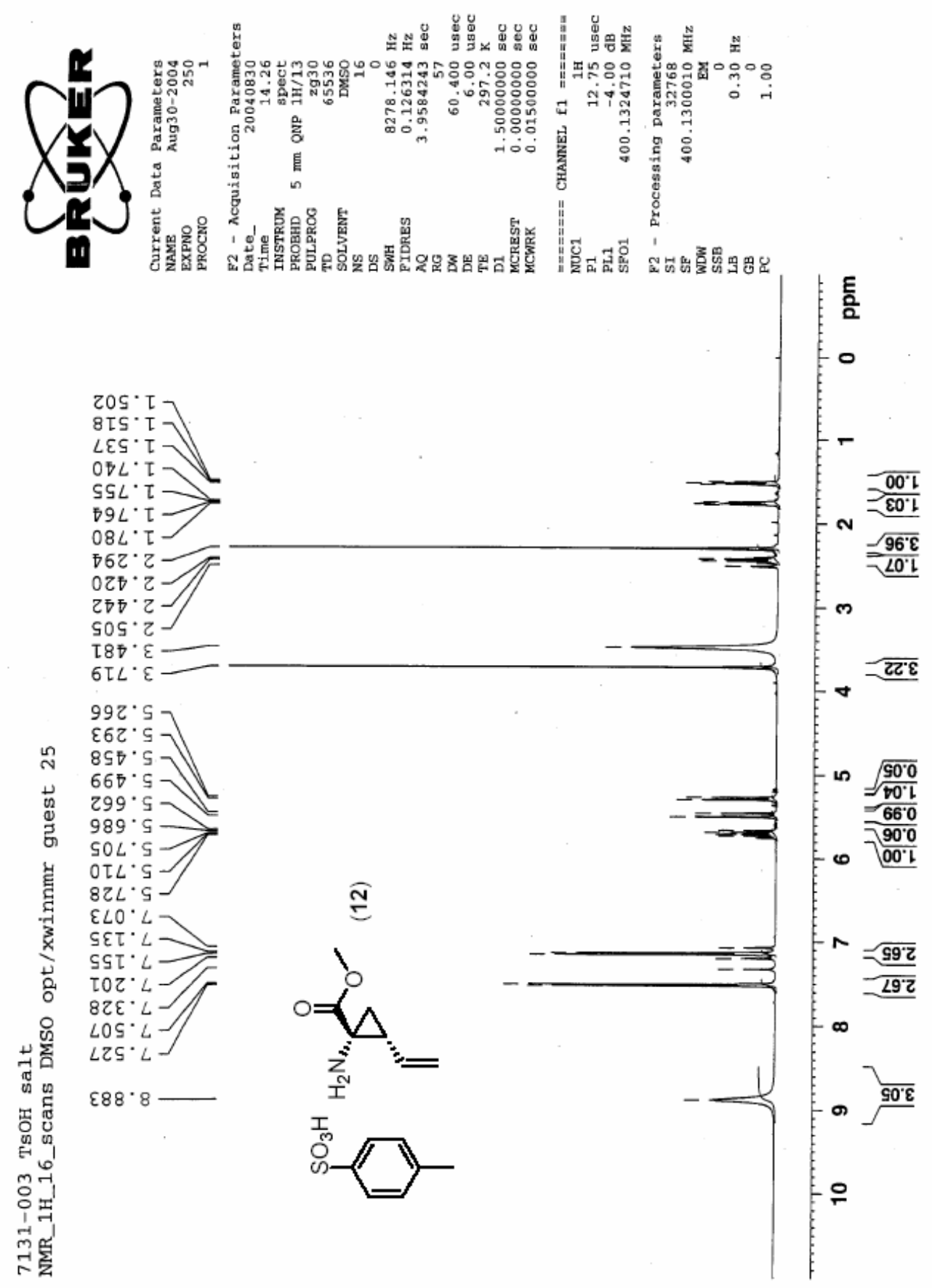


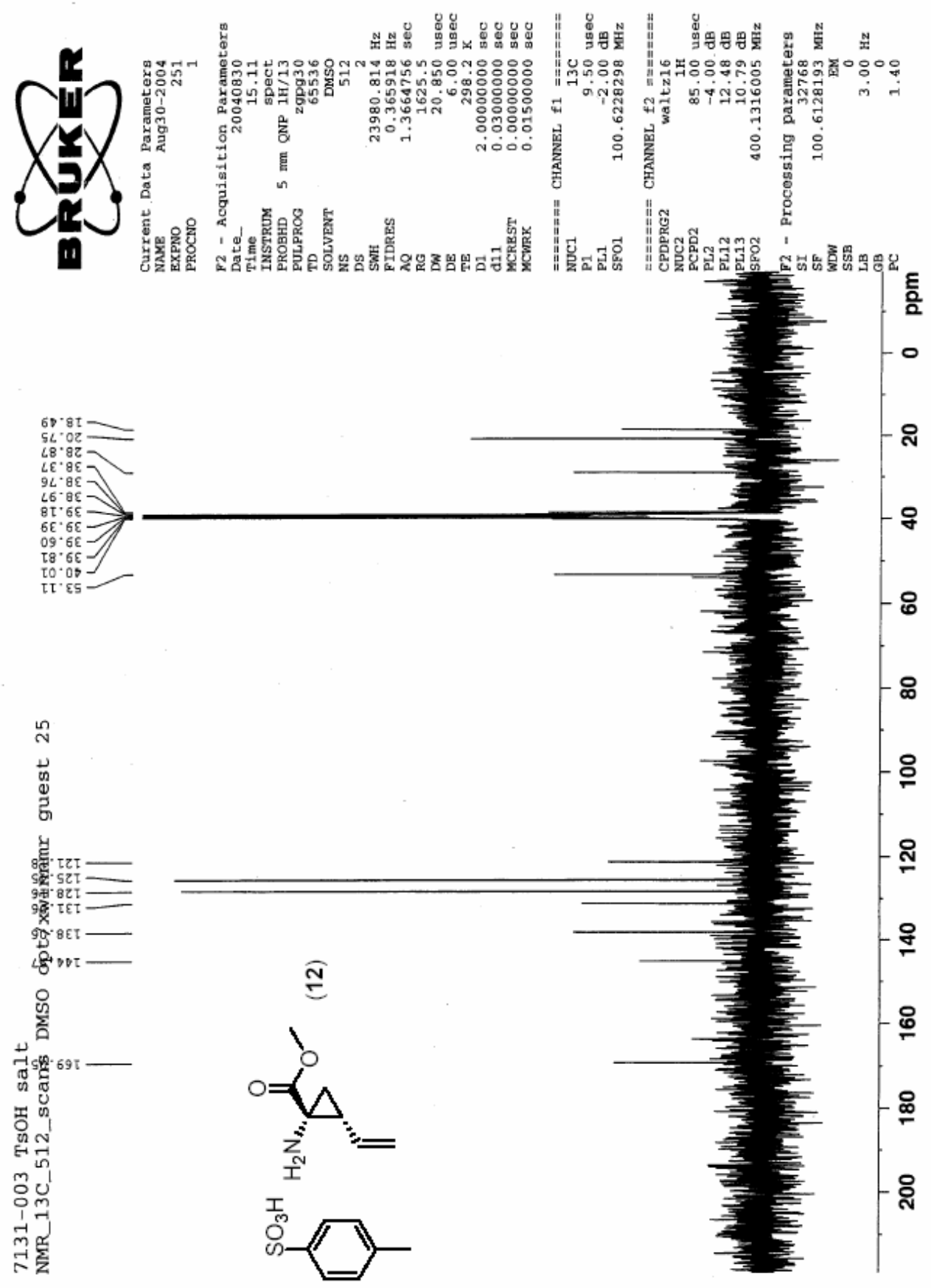



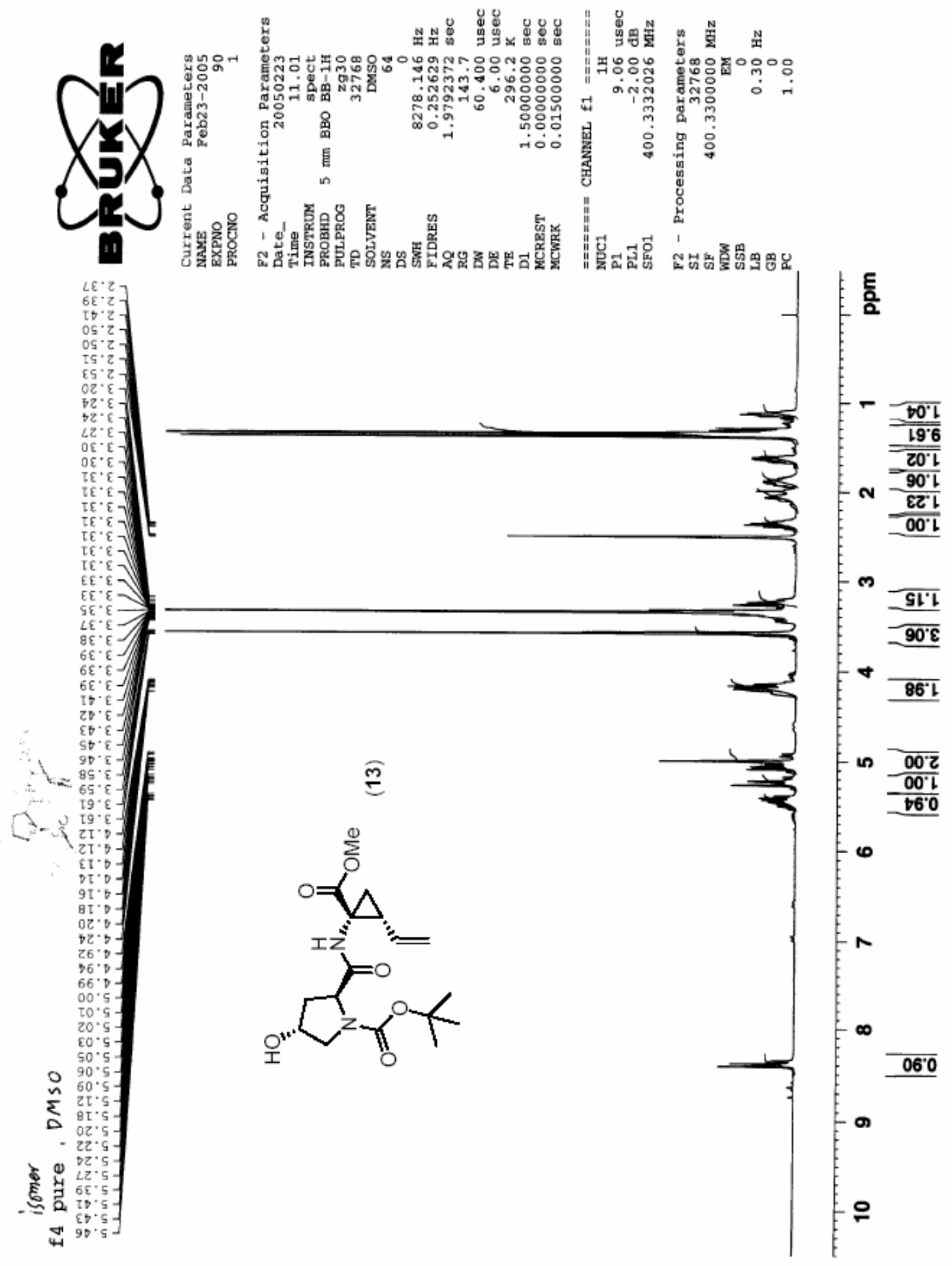

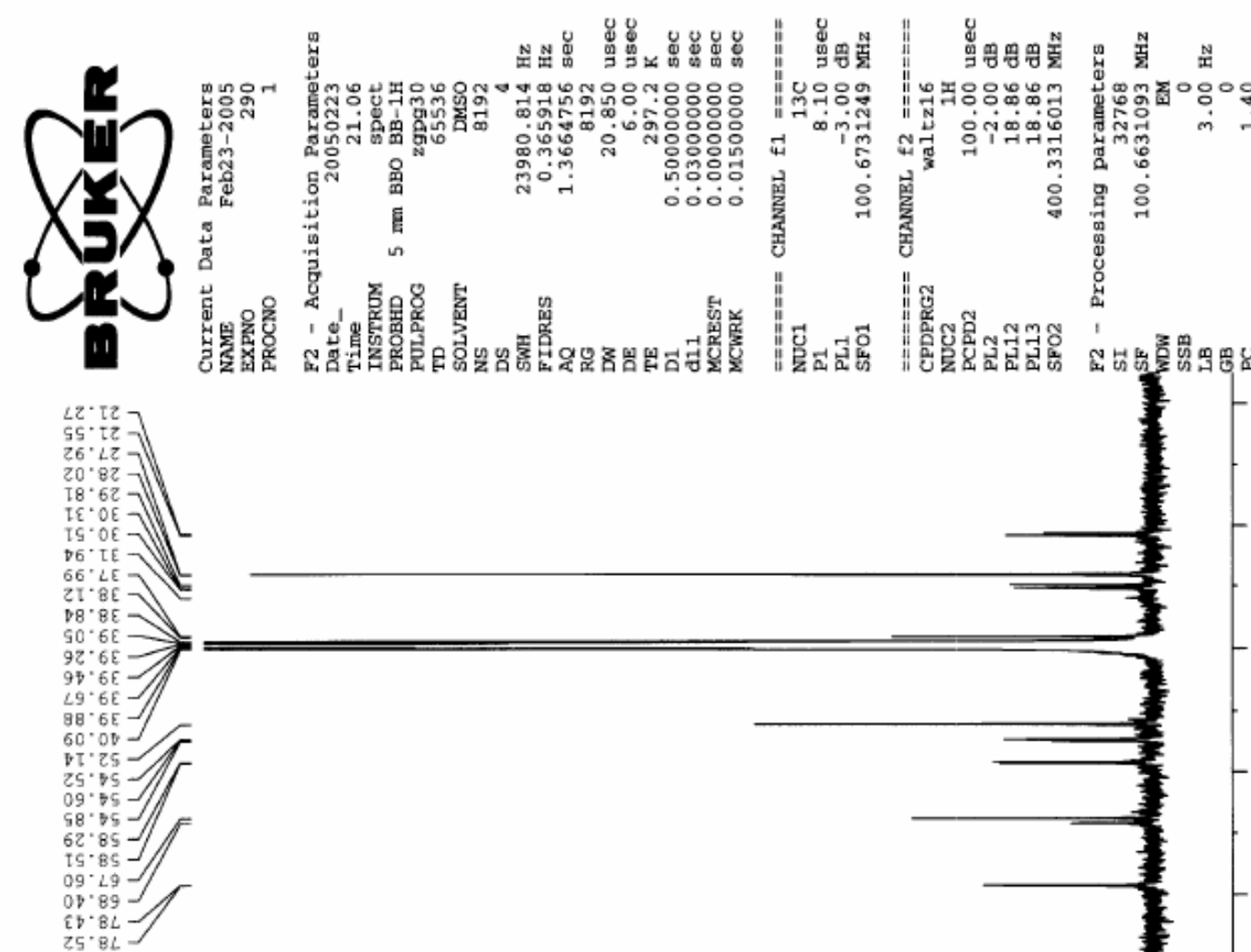

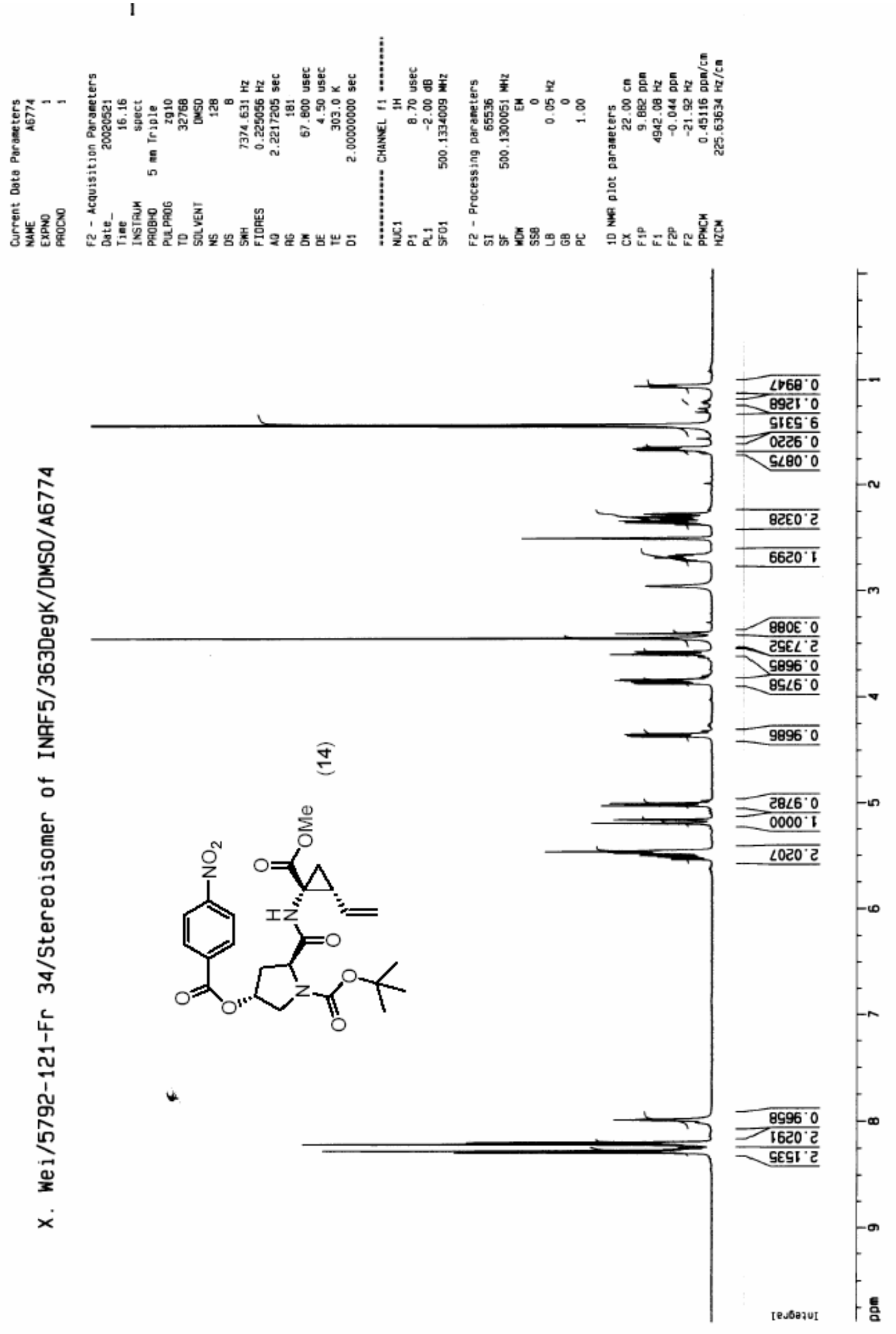


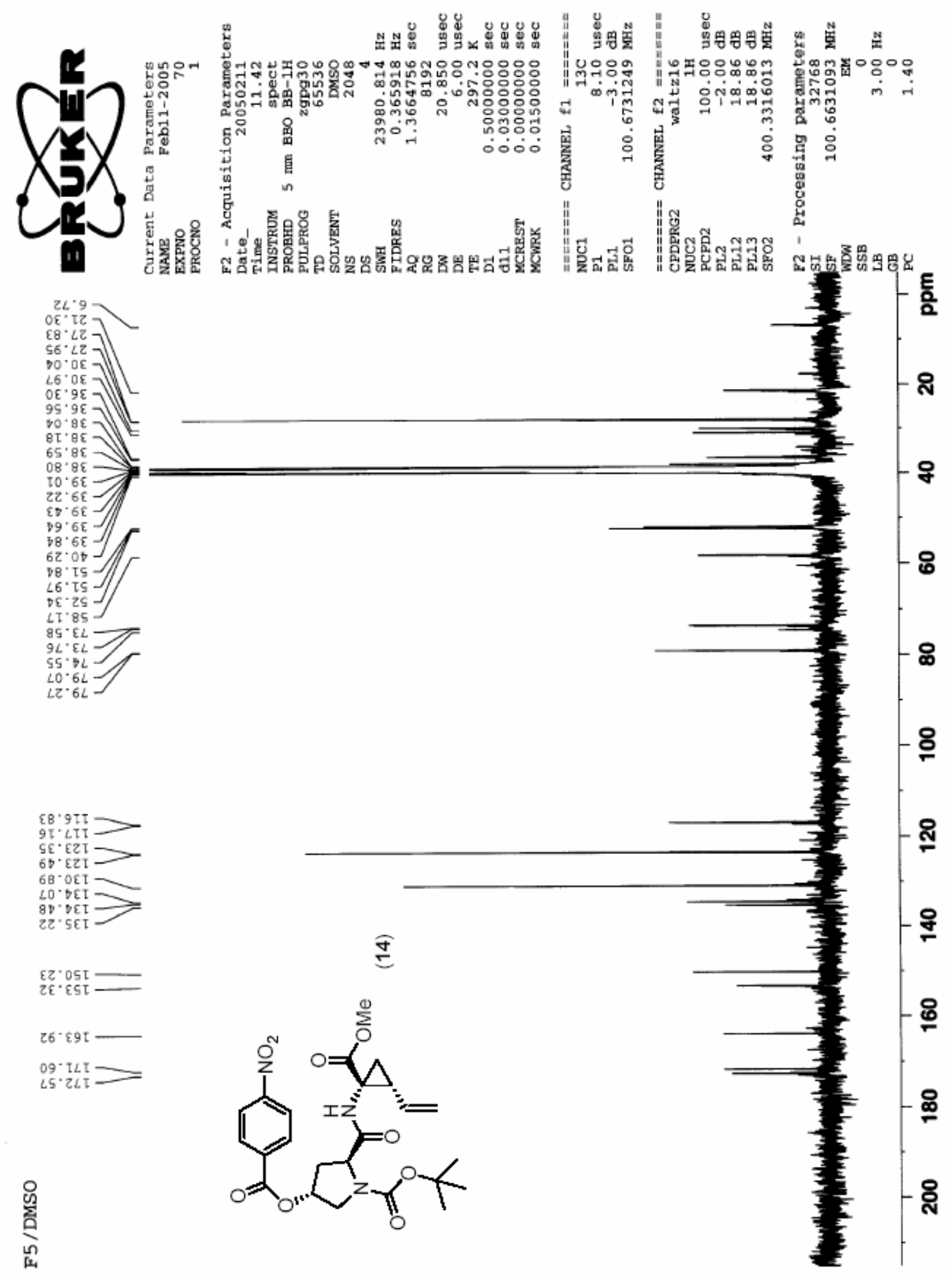



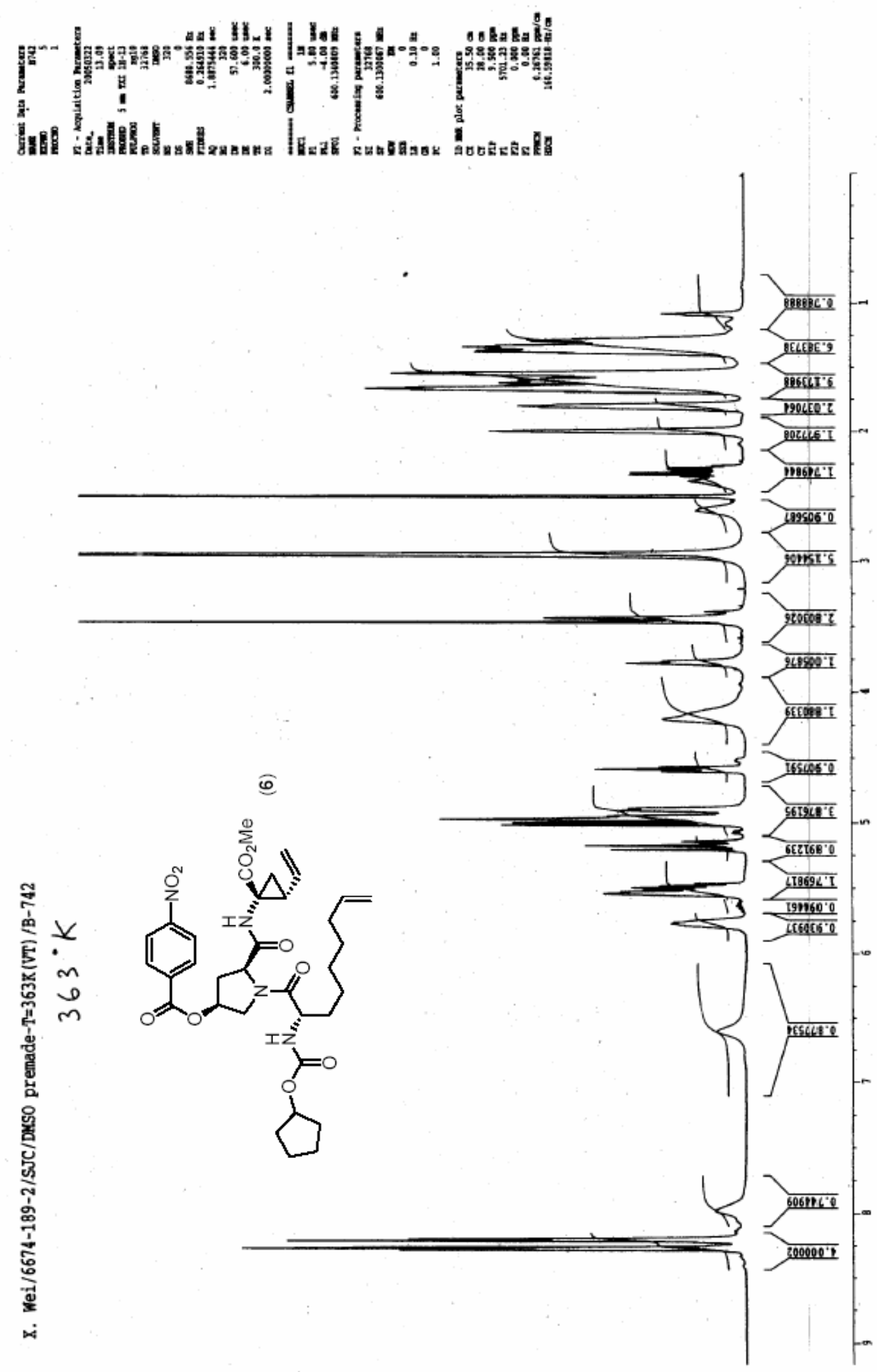

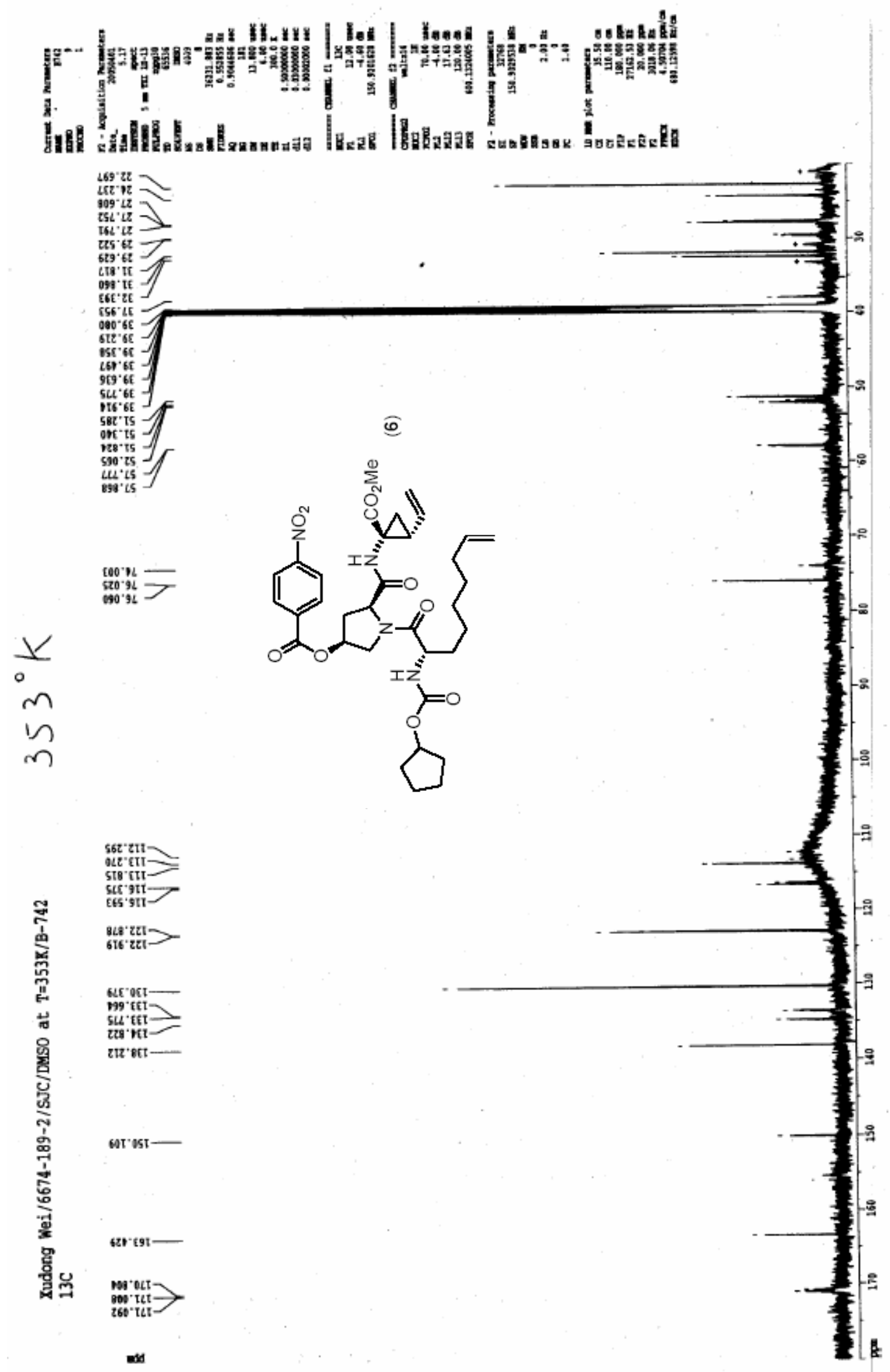

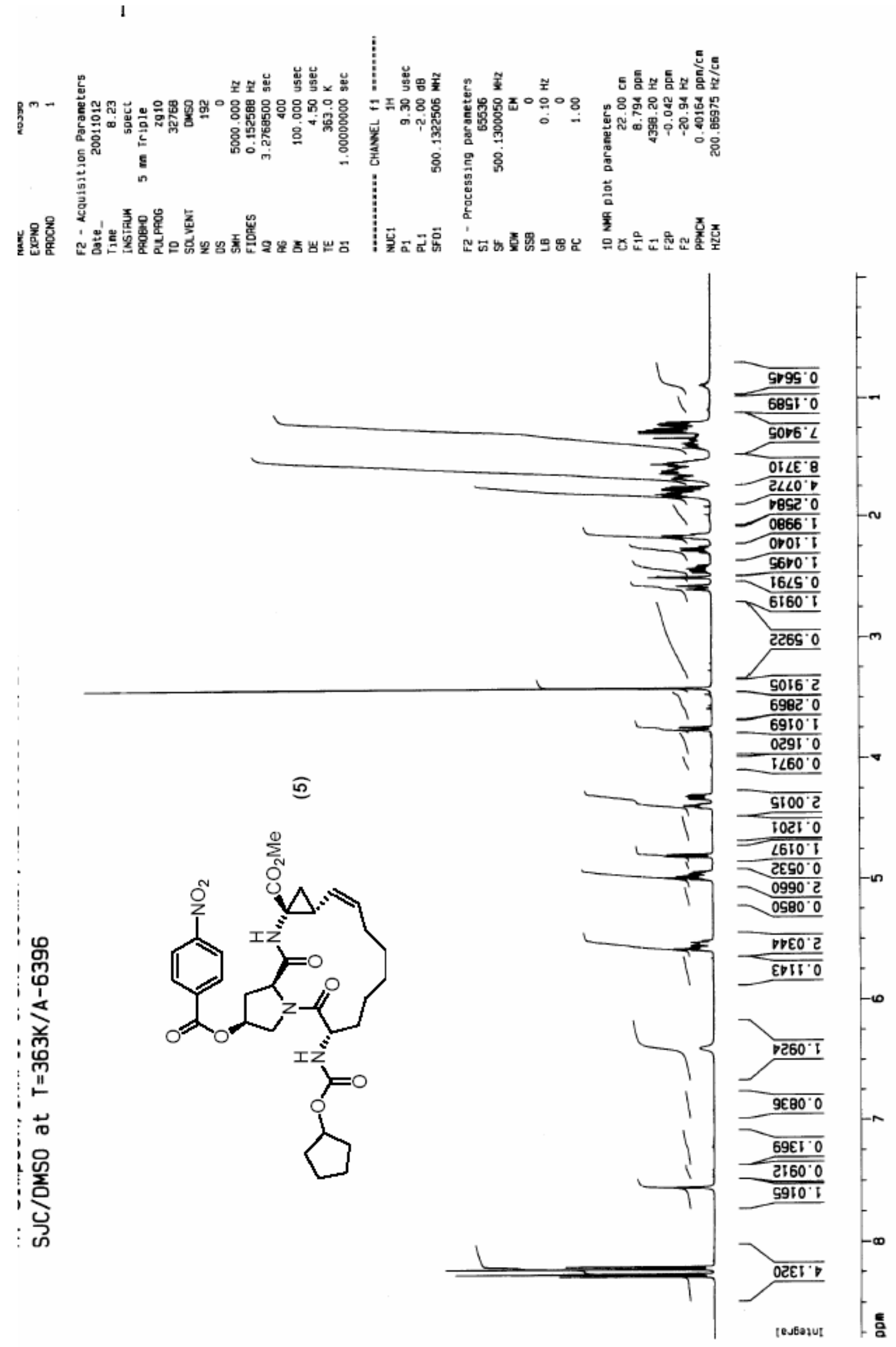


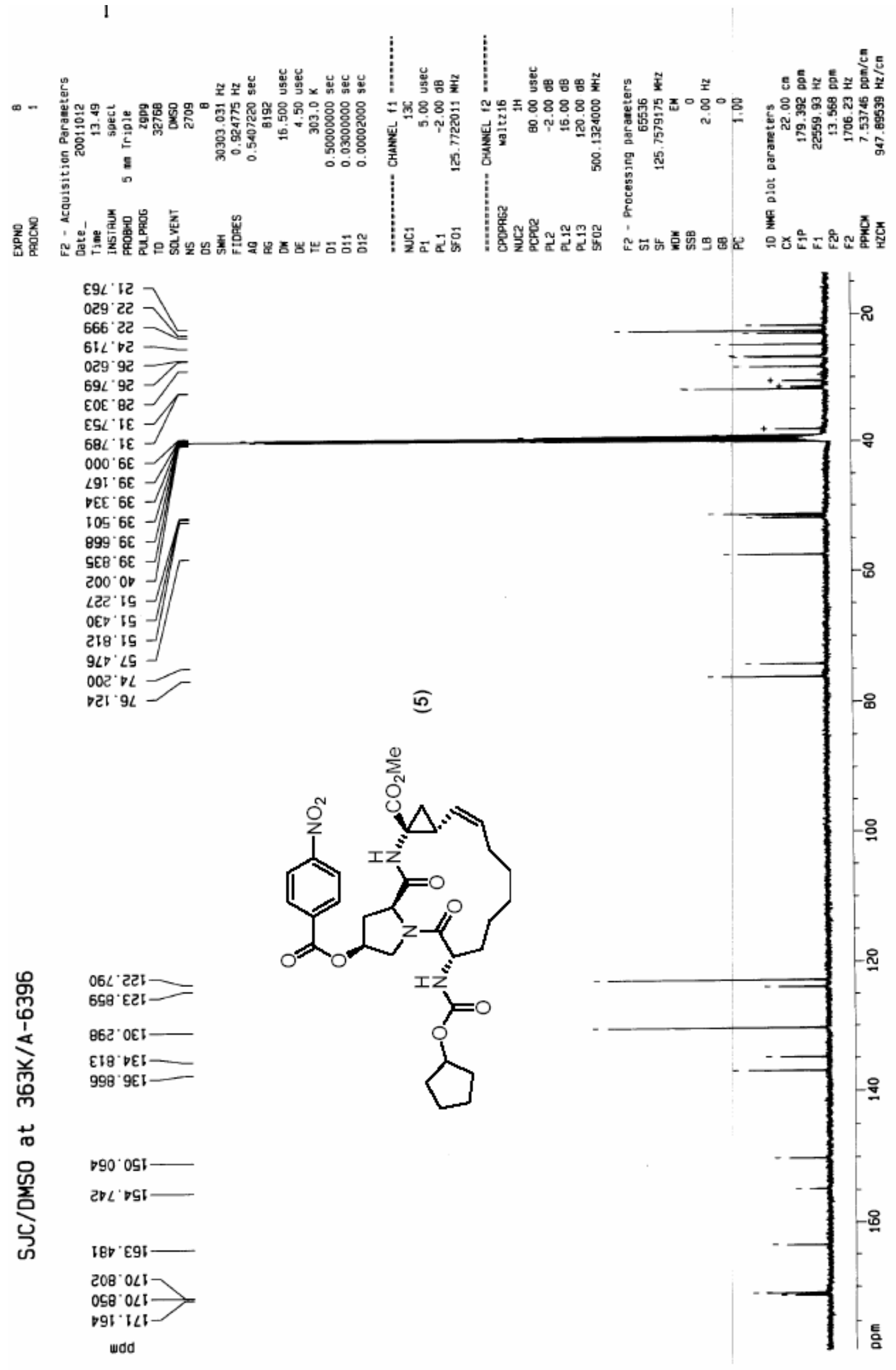



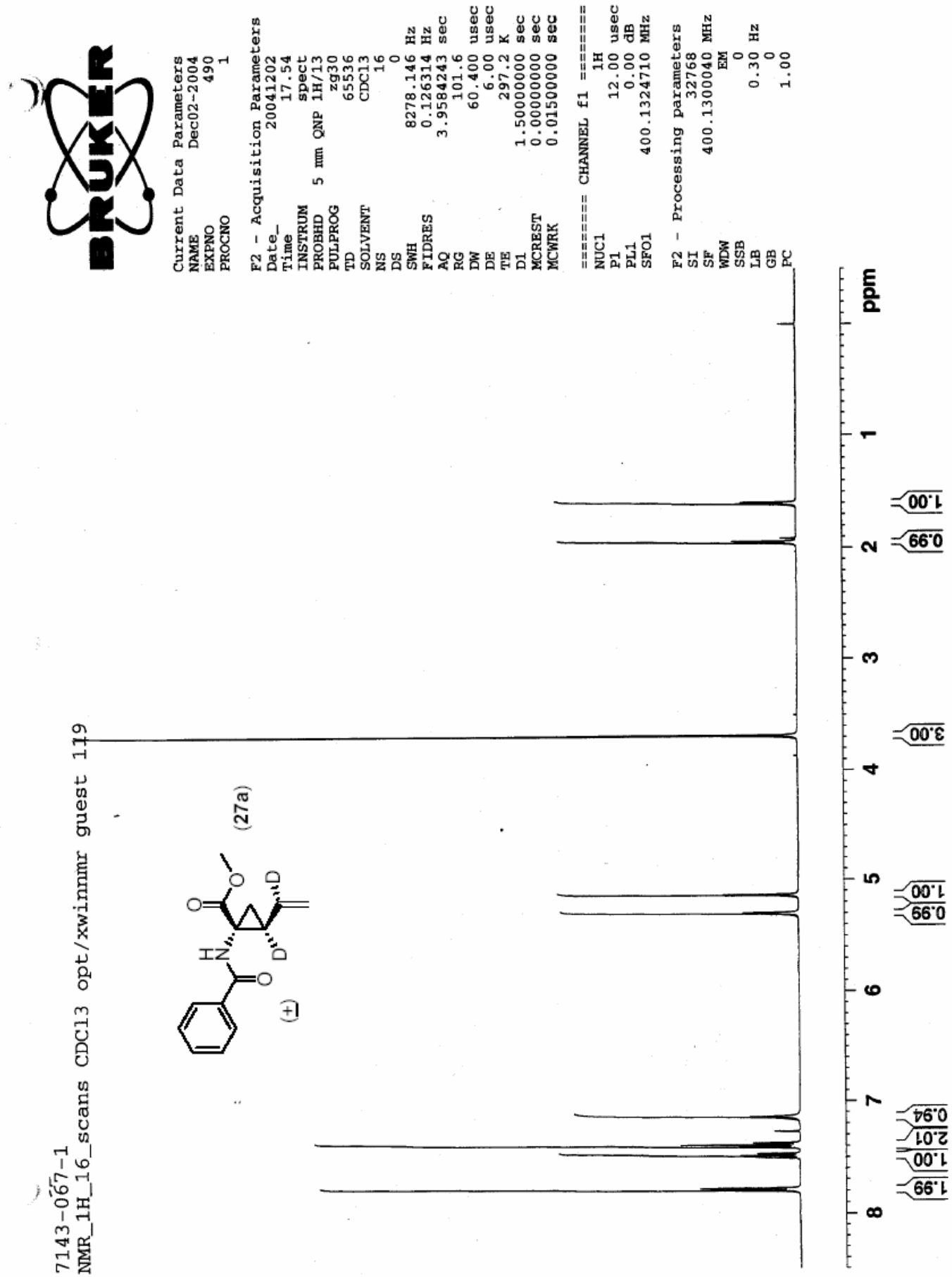

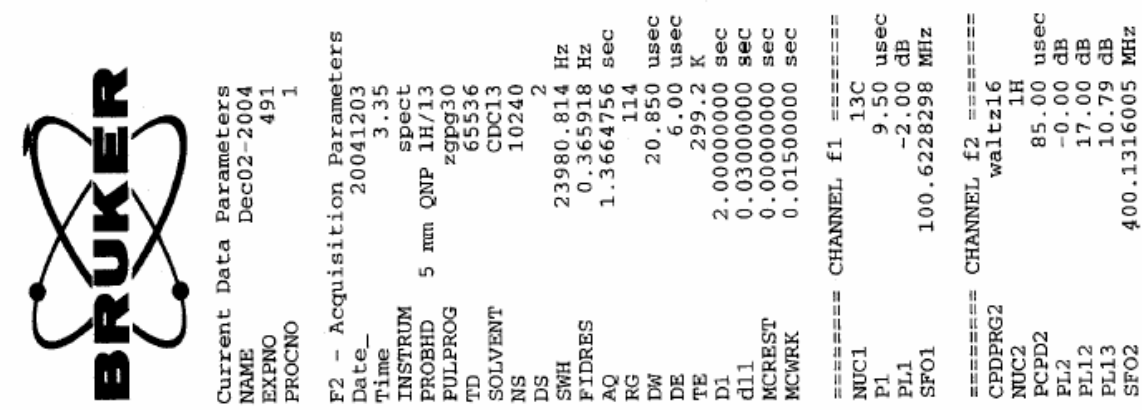

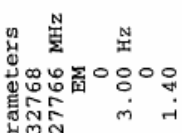

ปูलतु

울

LO'0-
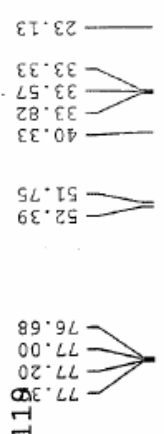

न

on

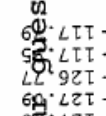

先. $\angle 2 T$

8ช $82 \tau$

唋. $62 \tau$

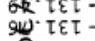

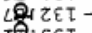

政 $\varepsilon \varepsilon \tau$

g. $\varepsilon \varepsilon \tau-$

要

90.89I

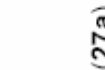

$\frac{1}{2}$

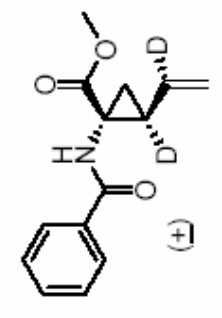

I
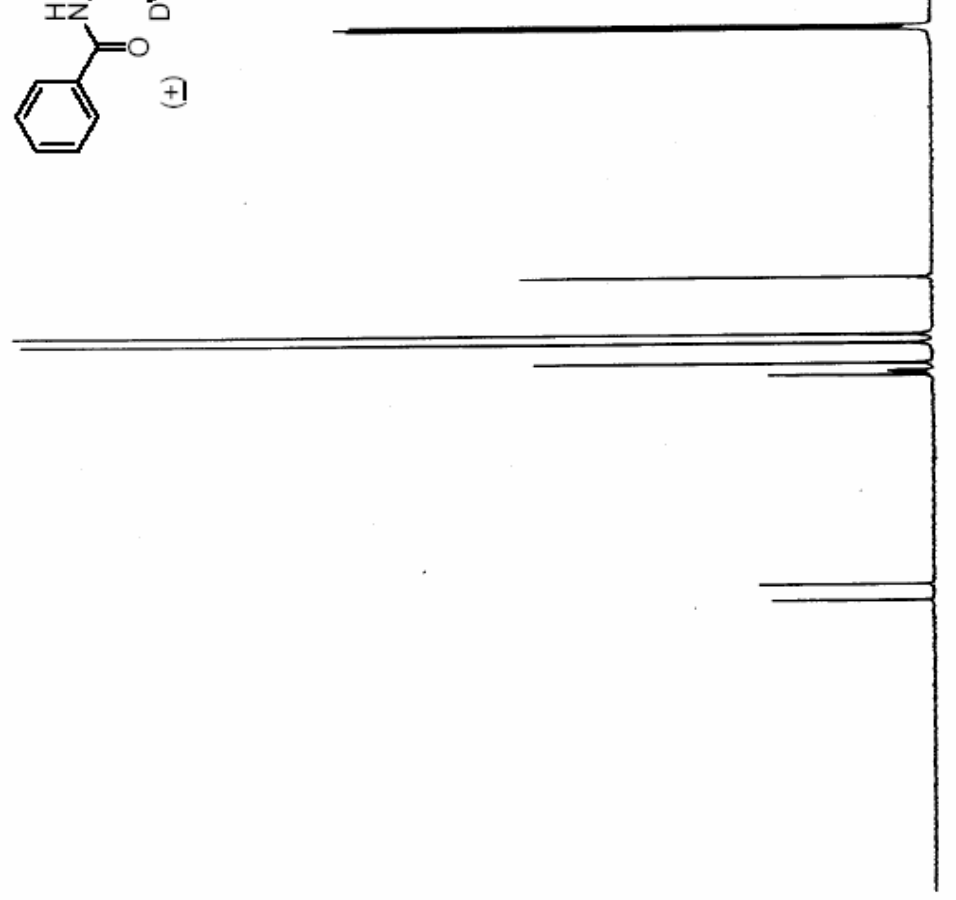

สิ

$\left.\right|_{0} ^{-\frac{2}{2}}$

02

교

150

i)

学啰 

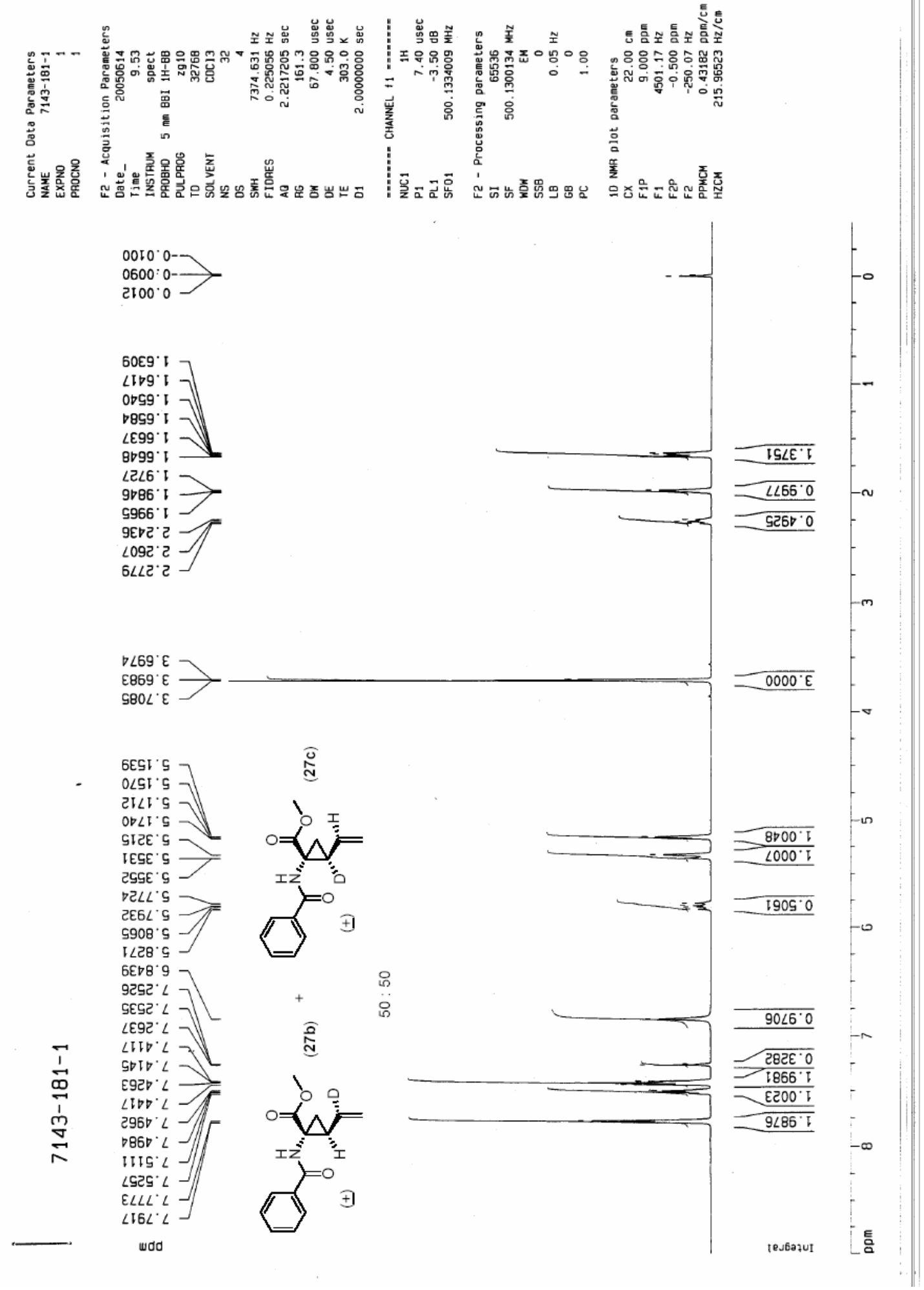


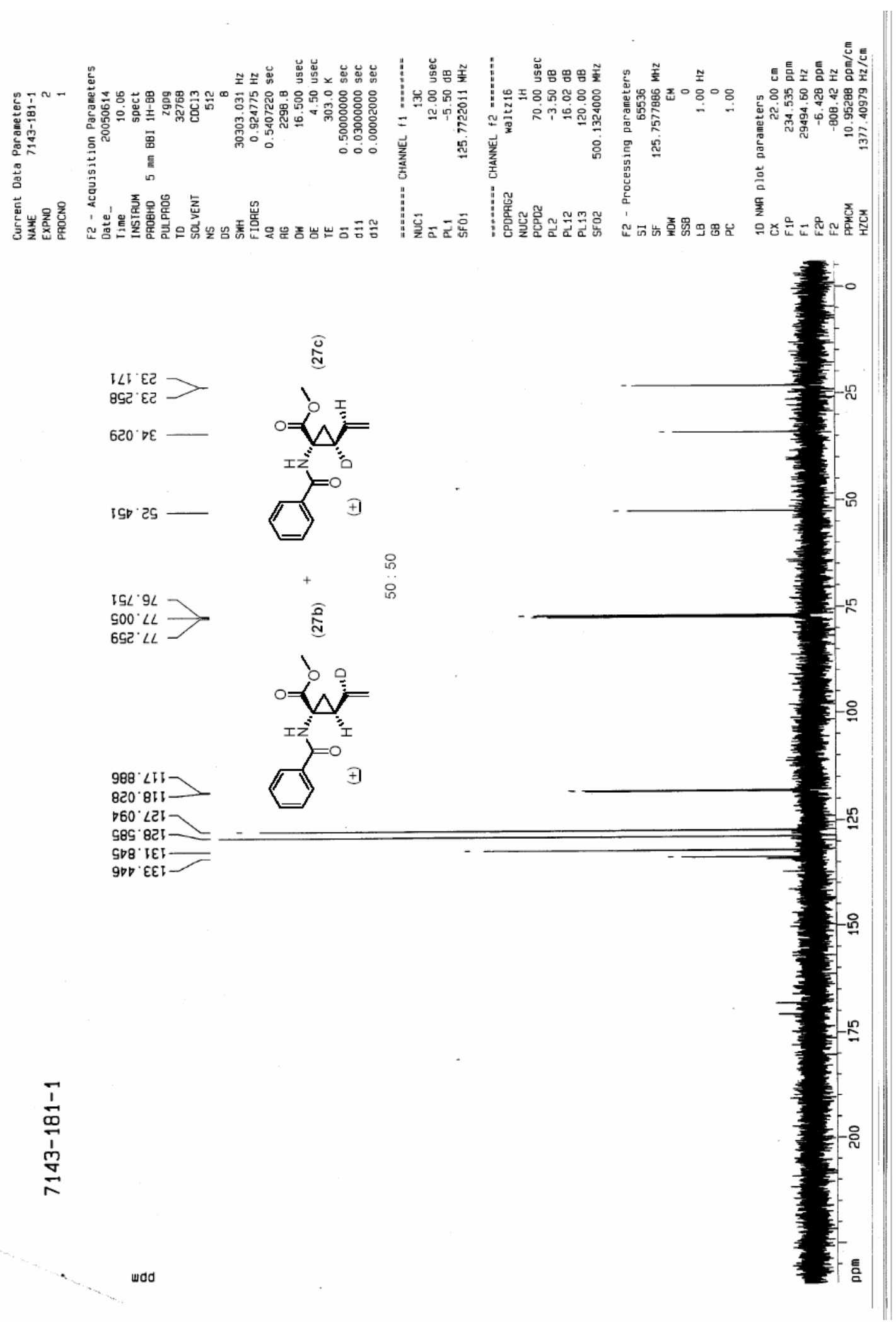



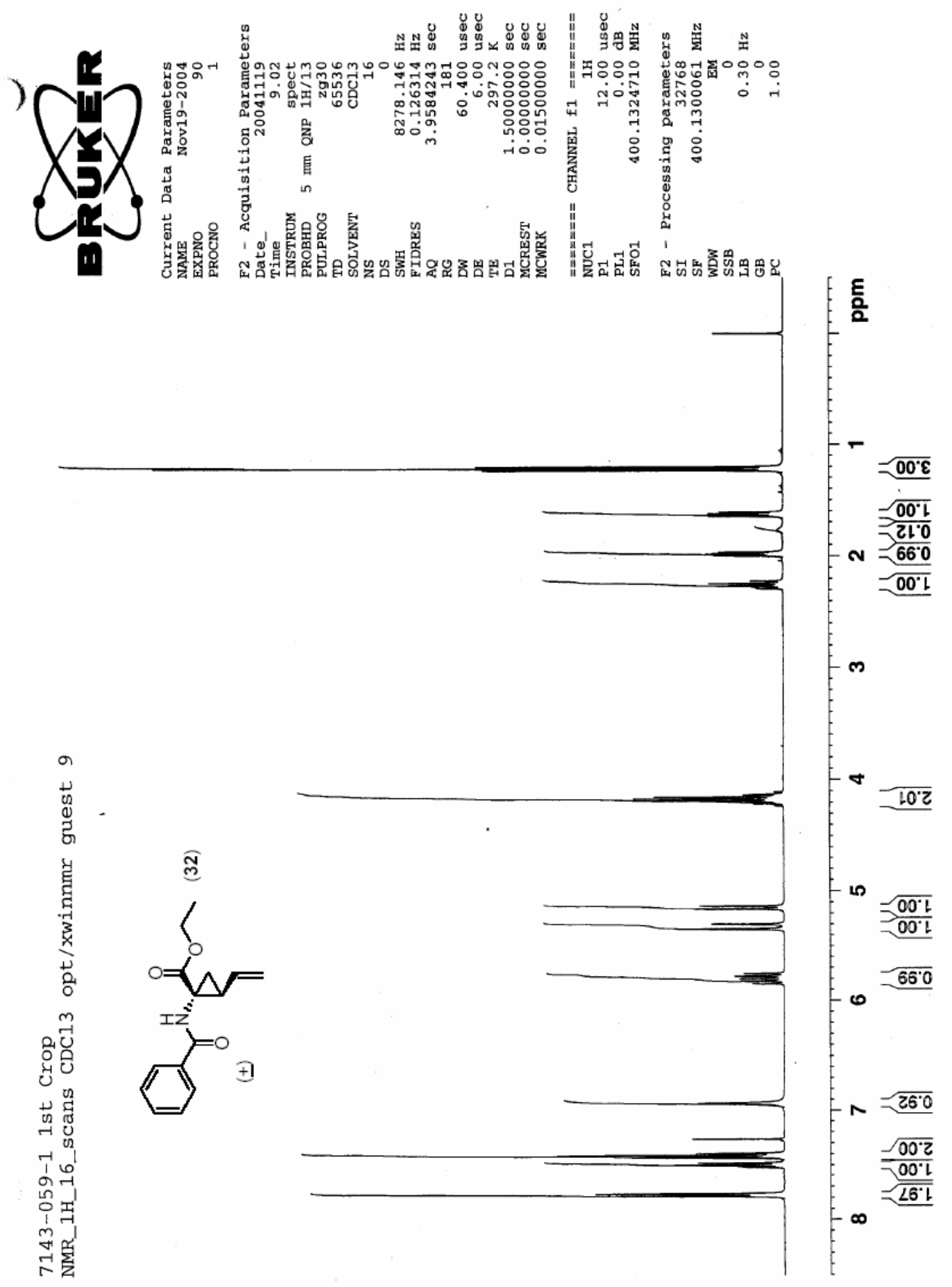


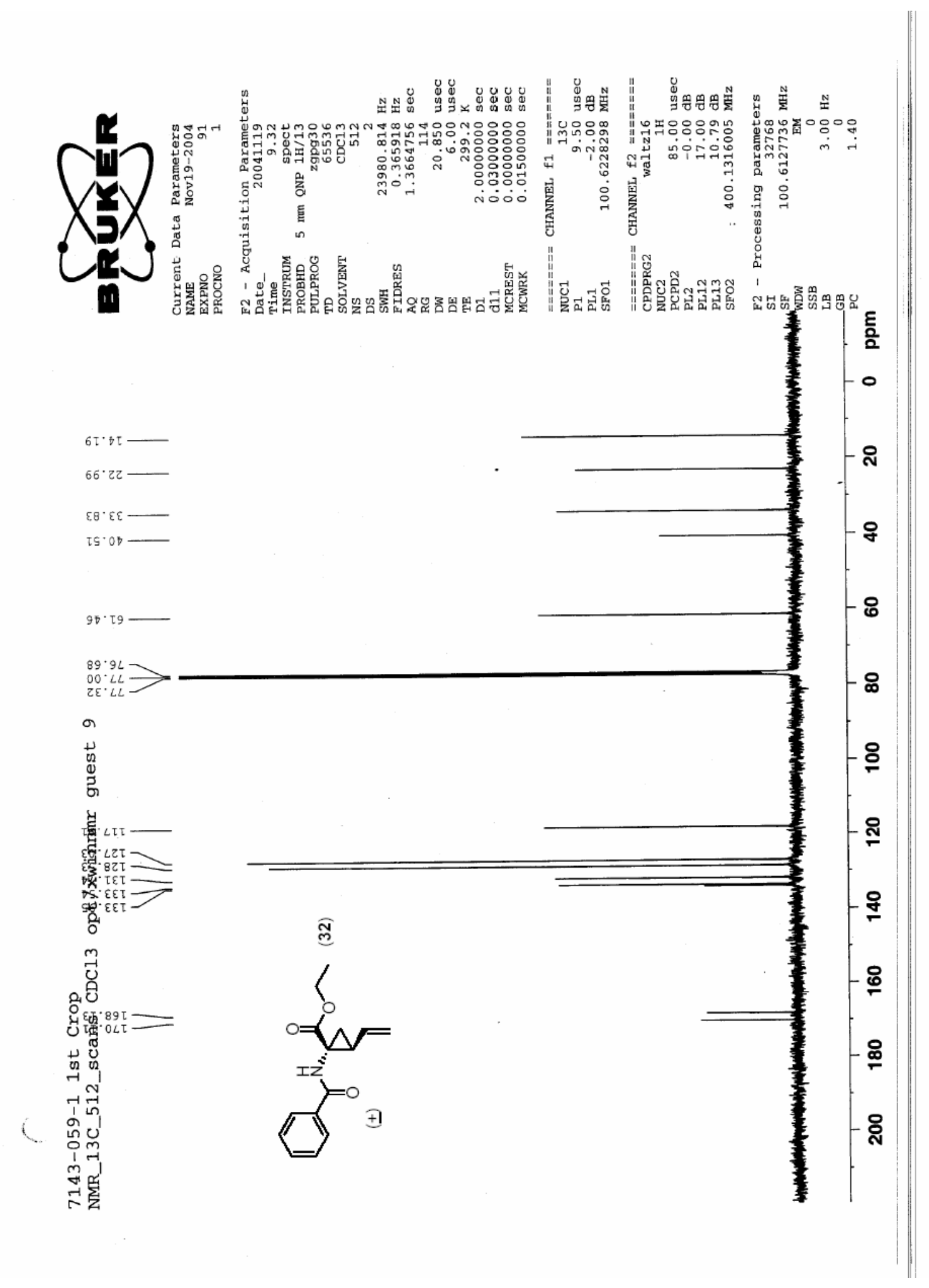

